\title{
Classical black hole scattering from a worldline quantum field theory
}

\author{
Gustav Mogull, ${ }^{a, b, c}$ Jan Plefka ${ }^{b}$ and Jan Steinhoff ${ }^{c}$ \\ ${ }^{a}$ Department of Physics and Astronomy, Uppsala University, \\ Box 516, 75108 Uppsala, Sweden \\ ${ }^{b}$ Institut für Physik und IRIS Adlershof, Humboldt-Universität zu Berlin, \\ Zum Großen Windkanal 6, D-12489 Berlin, Germany \\ ${ }^{c}$ Max-Planck-Institut für Gravitationsphysik (Albert-Einstein-Institut), \\ Mühlenberg 1, D-14476 Potsdam, Germany \\ E-mail: gustav.mogull@aei.mpg.de, jan.plefka@hu-berlin.de, \\ jan.steinhoff@aei.mpg.de
}

Abstract: A precise link is derived between scalar-graviton S-matrix elements and expectation values of operators in a worldline quantum field theory (WQFT), both used to describe classical scattering of black holes. The link is formally provided by a worldline path integral representation of the graviton-dressed scalar propagator, which may be inserted into a traditional definition of the S-matrix in terms of time-ordered correlators. To calculate expectation values in the WQFT a new set of Feynman rules is introduced which treats the gravitational field $h_{\mu \nu}(x)$ and position $x_{i}^{\mu}\left(\tau_{i}\right)$ of each black hole on equal footing. Using these both the 3PM three-body gravitational radiation $\left\langle h^{\mu \nu}(k)\right\rangle$ and 2PM two-body deflection $\Delta p_{i}^{\mu}$ from classical black hole scattering events are obtained. The latter can also be obtained from the eikonal phase of a $2 \rightarrow 2$ scalar S-matrix, which we show corresponds to the free energy of the WQFT.

KeYwords: Scattering Amplitudes, Black Holes, Classical Theories of Gravity

ArXiv EPrint: 2010.02865

In memory of Sissi Plefka and Jürgen Steinhoff 


\section{Contents}

1 Introduction 1

2 Worldline actions versus S-matrices 4

2.1 Worldline action 4

2.2 Dressed propagators in the Feynman-Schwinger representation 5

$\begin{array}{lll}2.3 & \text { From the S-matrix to the worldline } & 7\end{array}$

3 Graviton-dressed propagator for a massive scalar field 9

3.1 Momentum space representation 9

$\begin{array}{ll}3.2 & \text { Putting the scalar legs on shell } \\ & 11\end{array}$

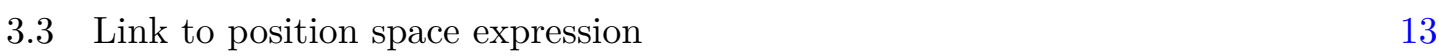

$\begin{array}{lll}3.4 & \text { Towards the eikonal phase } & 15\end{array}$

$\begin{array}{lll}3.5 & \text { Time-symmetric vs. retarded propagators } & 16\end{array}$

4 WQFT Feynman rules $\quad 16$

5 Radiation $\quad 20$

5.1 Two-body radiation (2PM) 21

5.2 Three-body radiation (3PM) 23

6 Deflections $\quad 25$

6.1 Leading order (1PM) 26

$\begin{array}{ll}6.2 & \text { Sub-leading order }(2 \mathrm{PM}) \\ \end{array}$

$\begin{array}{llr}7 & \text { Eikonal phase } & 28\end{array}$

8 Discussion $\quad 31$

A Derivation of the momentum space propagator 33

\section{Introduction}

Black holes are fascinating objects intimately tied to the fundamental properties of space, time and matter. Rightly they have been referred to as "the most perfect macroscopic objects in the universe" [1]. Their internal state is completely determined by their mass, charge and spin; in this respect they strongly resemble elementary particles, the equally fascinating constituents of matter and fundamental forces. These microscopic cousins of black holes are described using quantum field theory; their observables (such as cross sections) are derived from scattering amplitudes, which in turn have been called "the most perfect microscopic structures in the universe" [2]. 
With the advent of gravitational wave astronomy able to observe the binary inspirals and mergers of black holes and neutron stars [3-5], the need for high-precision theoretical predictions of their classical potentials and emerging gravitational radiation has arisen [6]. This is similar to the need for high-precision predictions of scattering cross section of elementary particles - a highly developed subject in quantum field theory. A number of complementary classical theoretical approaches to this central problem in general relativity have been established over recent years [7-12]. Taking up the parallelism with elementary particles, quantum field theoretical methods of perturbative quantum gravity have proven themselves very efficient for determining the classical gravitational interactions of black holes.

The gravitational two-body problem has traditionally been approached in a perturbative post-Newtonian (PN) weak-field and low-velocity approximation, where one simultaneously expands in powers of Newton's constant $G$ and in the relative velocity of the two bodies $v / c$ that are linked by the virial theorem for a bound system $\left(\frac{v^{2}}{c^{2}} \sim \frac{G m}{c^{2} r}\right)$. The non-relativistic general relativity formalism (NRGR) [13-15] uses an effective field theory (EFT) to model the massive bodies as point-like massive particles coupled to the gravitational field, and is valid for widely separated massive objects. Integrating out the suitably non-relativistically decomposed graviton field $h_{\mu \nu}(x)[16]$ in the path integral yields a Feynman diagrammatic expansion for the classical effective potential of the black holes and associated gravitational radiation - see e.g.refs. [17-21]. The suitably non-relativistically decomposed graviton field $h_{\mu \nu}(x)$ is integrated out in the path integral while the worldline trajectories of the black holes $x_{i}^{\mu}\left(\tau_{i}\right)$ are kept as classical background sources. The state of the art is $4 \mathrm{PN}$ level for the potential [22-32], parts of $5 \mathrm{PN}$ [33-36] and $6 \mathrm{PN}$ [37-42], and $3 \mathrm{PN}$ [43-45] for the gravitational radiation emitted from a quasi-circular inspiral (see also ref. [46]). Spin effects may also be taken into account [7, 8, 47, 48], in the conservative dynamics [49-51] (see refs. [52-58] for important early EFT work) and radiation [59-64].

Inspired by the progress made calculating scattering amplitudes, approaches involving a post-Minkowskian (PM) expansion in Newton's constant, which re-sum the entire PN expansion in velocity, have recently been gaining prominence. For instance, the worldline EFT may also be deployed in a PM weak-field scenario as one may naturally use perturbative quantum gravity to represent gravitons as a metric fluctuation about flat Minkowskian space-time. This is also the right approximation for black hole scattering events or $N$-body interaction scenarios. A worldline EFT formalism for the PM expansion was recently established in ref. [65] for conservative binary dynamics (including tidal effects, see also refs. [66-69]), and has now been successfully applied to order 3PM $\left(\mathcal{O}\left(G^{3}\right)\right)$ [70]. Earlier worldline-based PM calculations can be found in refs. [11, 12, 71-73] for the conservative sector, in refs. [74, 75] for radiation, and in refs. [76-78] for spin effects.

A fruitful alternative approach to capture the classical interactions of massive bodies in gravity has also been explored through a more direct examination of scattering amplitudes in perturbatively quantized gravity. While there are early works on the subject [79-81], this approach has blossomed in recent years upon employing modern on-shell methods for scattering amplitudes [82-86]. These works have led us to the $2 \mathrm{PM}[85,87,88]$ and 3PM $[38,89,90]$ results for the effective gravitational potential, as well as early results 
including spin effects [91-97]. The computational method established so far is somewhat intricate: starting from the scattering amplitude of two massive flavored scalar particles minimally coupled to gravity, and taking a subtle classical limit [86, 98], one matches the amplitudes obtained to those of an EFT of non-relativistic scalar particles in order to determine its conservative two-body potential [87, 99]. The so-obtained effective potential is then used to compute observables such as the scattering angle or the periastron advance in the bound system $[65,100,101]$.

Both approaches - involving the worldline EFT and modern scattering amplitudes agree on the final results for observables and conservative potentials in the PM expansion; the question of efficiency is a matter of debate (and taste). What has remained unclear, however, is whether there is a more direct connection between the amplitude and worldline EFT approaches. The present work fills this gap.

Our key observation is that the Feynman-Schwinger or worldline representation of the graviton-dressed scalar propagator $[102,103]$ provides this link. Inserting it into a timeordered correlation function of scalars and gravitons yields a precise map to expectation values of operators in a worldline quantum field theory (WQFT). This WQFT is the same worldline EFT discussed above [13-15, 65], but with the important additional ingredient that the worldline trajectories are also quantized. We write

$$
x^{\mu}(\tau)=b^{\mu}+\tau v^{\mu}+z^{\mu}(\tau)
$$

where $z^{\mu}$ describes the perturbation of a black hole from its original straight-line trajectory in a binary scattering process, and integrate out $z^{\mu}$ in the WQFT path integral together with the graviton $h_{\mu \nu}$. So both the worldline and the graviton field are treated on an equal footing in our approach.

Previous results for expressing observables of the black holes (such as their deflections and radiation) encountered through scattering amplitudes derived in ref. [86] follow elegantly from correlators in our WQFT. The tedious procedure in the traditional worldline EFT approach of first finding the effective potential by integrating out the graviton and thereafter solving the resulting equations of motion in terms of a perturbative ansatz for the $z^{\mu}$ of eq. (1.1) - is streamlined through WQFT Feynman rules, which provide a fast track to the integrands yielding the observables. The classical eikonal of the scattering of two massive particles, encoding the classical part of the 4-point amplitude, can be calculated directly from the WQFT. ${ }^{1}$ So we expect our new formalism to not only be of foundational interest in clarifying the connection between scattering amplitudes and the worldline theory, but also to be of calculational advantage for precision calculations in the classical gravity two-body problem. We demonstrate this by establishing the sub-leading corrections to the deflection and radiation, the latter having not appeared in the literature.

The rest of our paper is organized as follows. In section 2 we introduce the FeynmanSchwinger representation of the gravitationally dressed scalar propagator, and demonstrate how it may be inserted into time-ordered correlation functions. Then in section 3 we

\footnotetext{
${ }^{1}$ Interestingly, a similar connection was indeed studied a long time ago in ref. [104] recovering the eikonal result $[105,106]$ for the ultra-relativistic limit of a string scattering computation.
} 
explain how to move from correlators to S-matrices, by cutting the propagators of external legs. We also begin our discussion of the eikonal phase of scalar scattering, demonstrating that it corresponds precisely to the free energy of the WQFT. In section 4 we introduce Feynman rules for the WQFT, which we can use to conveniently calculate expectation values in Fourier space. Using these, in sections 5 and 6 we compute the $2 \mathrm{PM}$ radiation $k^{2}\left\langle h^{\mu \nu}(k)\right\rangle$ integrand and deflection $\Delta p_{1}^{\mu}$ from an inelastic scattering of two black holes respectively, drawing a close comparison with the equivalent amplitudes-based calculations. In section 5 we also compute the 3PM radiation integrand from an inelastic scattering of three black holes. Finally, in section 7 we revisit the eikonal phase and demonstrate how useful observables, including the deflection and scattering angle, can be obtained from it. In section 8 we conclude.

\section{Worldline actions versus S-matrices}

In this section we show how expectation values of operators in a worldline theory, corresponding to gravitational observables, can be directly obtained from S-matrices in the classical limit. The link is formally provided by a worldline representation of the massive scalar propagator in a fixed gravitational background, which we refer to as the FeynmanSchwinger form. First we rewrite the worldline action.

\subsection{Worldline action}

We seek to describe the scattering of two (or more) unbound black holes. The spinless black holes may be described in an effective field theory (EFT) framework [13] as relativistic massive particles moving along their worldlines and coupled to gravity:

$$
S=S_{\mathrm{EH}}+S_{\mathrm{gf}}+\sum_{i} S_{\mathrm{pm}}^{(i)} .
$$

$S_{\mathrm{EH}}$ is the usual Einstein-Hilbert action (working in $D$ dimensions):

$$
S_{\mathrm{EH}}=-2 m_{\mathrm{Pl}}^{D-2} \int \mathrm{d}^{D} x \sqrt{-g} R .
$$

Using the weak field approximation we expand

$$
g_{\mu \nu}=\eta_{\mu \nu}+\kappa h_{\mu \nu}
$$

where $\kappa=m_{\mathrm{Pl}}^{1-D / 2}$, thereafter raising and lowering indices with the "mostly minus" Minkowski metric $\eta_{\mu \nu}=\operatorname{diag}(+1,-1,-1,-1)$. Our gauge-fixing term $S_{\mathrm{gf}}$ is

$$
S_{\text {gf }}=\int \mathrm{d}^{D} x\left(\partial_{\nu} h^{\mu \nu}-\frac{1}{2} \partial^{\mu} h^{\nu}{ }_{\nu}\right)^{2}
$$

which imposes the usual de Donder gauge condition $\partial_{\nu} h^{\mu \nu}=\frac{1}{2} \partial^{\mu} h^{\nu}{ }_{\nu}$.

The point mass action for a single extended object (such as a black hole) moving along a worldline $x^{\mu}(\tau)$ and with proper time $\mathrm{d} \tau=\sqrt{g_{\mu \nu} d x^{\mu} d x^{\nu}}$ reads

$$
S_{\mathrm{pm}}=-m \int \mathrm{d} \tau+c_{R} \int \mathrm{d} \tau R(x)+c_{V} \int \mathrm{d} \tau R_{\mu \nu}(x) \dot{x}^{\mu} \dot{x}^{\nu}+\ldots
$$


The first term induces geodesic motion with respect to the metric $g_{\mu \nu}$. In addition, we allow for non-minimal couplings of the point mass to the gravitational field parametrized by a priori unknown Wilson coefficients $c_{R / V}$. There is an infinite number of terms beyond these two organized in higher powers of the curvature tensor and derivatives. These terms account for the internal structure of the extended object to be described. The first two leading terms above do not contribute to physical observables as they may may be removed by a (singular) field redefinition of $h_{\mu \nu}$, as was argued ref. [13] and subsequently demonstrated at $4 \mathrm{PN}$ in ref. [28] (see also ref. [20]). We shall drop them for the time being, yet the $c_{R}$ term will have a role to play shortly.

The point-mass action can also be written in a Polyakov form:

$$
S_{\mathrm{pm}}=-\frac{m}{2} \int_{-\infty}^{\infty} \mathrm{d} \tau\left(e^{-1} g_{\mu \nu} \dot{x}^{\mu} \dot{x}^{\nu}+e\right),
$$

where $e(\tau)$ is the einbein with equation of motion $e^{2}=g_{\mu \nu} \dot{x}^{\mu} \dot{x}^{\nu}$. Solving for $e(\tau)$ we recover the point-mass action in eq. (2.5); alternatively, we can gauge fix $e=1$ to enforce $g_{\mu \nu} \dot{x}^{\mu} \dot{x}^{\nu}=1$, which identifies $\tau$ as the proper time. In the latter case the point-mass action becomes

$$
S_{\mathrm{pm}}=-\frac{m}{2} \int_{-\infty}^{\infty} \mathrm{d} \tau\left(g_{\mu \nu} \dot{x}^{\mu} \dot{x}^{\nu}+1\right)
$$

This form of the particle action is superior to the initial one eq. (2.5) as it does not involve any square roots and only displays a linear coupling of the worldline to the graviton field $h_{\mu \nu}$ in the weak-field expansion.

\subsection{Dressed propagators in the Feynman-Schwinger representation}

Next we consider a massive complex scalar field $\phi(x)$ coupled to Einstein gravity as the QFT avatar of a single black hole. For a binary system one simply generalizes to two differently flavored massive scalars $\phi_{i}(x)$. The relevant action reads

$$
\begin{aligned}
S^{\prime} & =S_{\mathrm{EH}}+S_{\mathrm{gf}}+\sum_{i=1}^{2} S_{i}, \\
\text { with } \quad S_{i} & =\int \mathrm{d}^{D} x \sqrt{-g}\left(g^{\mu \nu} \partial_{\mu} \phi_{i}^{\dagger} \partial_{\nu} \phi_{i}-m_{i}^{2} \phi_{i}^{\dagger} \phi_{i}-\xi R \phi_{i}^{\dagger} \phi_{i}\right),
\end{aligned}
$$

where we allow for a non-minimal coupling of the scalar field to the background curvature controlled by the dimensionless parameter $\xi$. In a fixed gravitational background the associated Green's function $G\left(x, x^{\prime}\right)$ of the scalar field obeys the partial differential equation

$$
\left(\nabla_{\mu} \nabla^{\mu}+m^{2}+\xi R\right) G\left(x, x^{\prime}\right)=\sqrt{-g} \delta^{(D)}\left(x-x^{\prime}\right),
$$

where $\nabla_{\mu}$ denotes the gravitational covariant derivative, i.e. $\nabla_{\mu} \nabla^{\mu} G=\partial_{\mu} \partial^{\mu} G+\Gamma^{\mu}{ }_{\mu \nu} \partial^{\nu} G$. There exists a worldline path integral representation for $G\left(x, x^{\prime}\right)$ that we shall now review.

Let us first consider the analogous situation in scalar QED. The Green's function $G_{A}\left(x, x^{\prime}\right)=\left\langle\Omega\left|T\left\{\phi(x) \phi^{\dagger}\left(x^{\prime}\right)\right\}\right| \Omega\right\rangle$ for a massive charged scalar propagating in an electromagnetic background $A_{\mu}(x)$ obeys

$$
\left(D_{\mu} D^{\mu}+m^{2}\right) G_{A}\left(x, x^{\prime}\right)=\delta^{(D)}\left(x-x^{\prime}\right)
$$


with $D_{\mu}=\partial_{\mu}+i e A_{\mu}$. It was first proposed by Feynman [107] in the birth phase of QED that this Green's function has a worldline path integral representation ${ }^{2}$

$$
G_{A}\left(x, x^{\prime}\right)=\int_{0}^{\infty} \mathrm{d} s e^{-i s m^{2}} \int_{x(0)=x}^{x(s)=x^{\prime}} D[x] \exp \left[-i \int_{0}^{s} \mathrm{~d} \sigma\left(\frac{1}{4} \eta_{\mu \nu} \frac{\mathrm{d} x^{\mu}}{\mathrm{d} \sigma} \frac{\mathrm{d} x^{\nu}}{\mathrm{d} \sigma}+e A_{\mu} \dot{x}^{\mu}\right)\right],
$$

which reduces to the Schwinger proper time representation of the propagator in the free $(e=0)$ case. Notice that $\sigma$ (and therefore $s$ ) has dimensions of $m^{-2}$, so we distinguish it from the proper time $\tau$ with dimensions of $m^{-1}$.

This worldline representation of the photon-dressed propagator - which we refer to as the Feynman-Schwinger representation - is very efficient for computing effective actions at one-loop order, e.g. to compute the Euler-Heisenberg action in the case of constant electromagnetic field strengths. The generalization to the non-abelian case is straightforward: simply insert a trace over color states in the path integrand and replace the gauge field by $A_{\mu}^{a} T^{a}$ with the generators $T^{a}$ in the representation of the scalar. This representation of the Green's function has been used for efficient calculations of one-loop amplitudes and effective actions in gauge theories [109], and it also arises through the point particle limit of open strings [110] — see refs. [111, 112] for comprehensive reviews.

In gravity the problem is more intricate and subject to a longer discussion in the literature. Naively one would expect to simply generalize eq. (2.11) to a curved background upon promoting $\eta_{\mu \nu}$ to $g_{\mu \nu}$, plus including possible curvature couplings:

$$
G\left(x, x^{\prime}\right) \sim \int_{0}^{\infty} \mathrm{d} s e^{-i s m^{2}} \int_{x(0)=x}^{x(s)=x^{\prime}} \mathcal{D}[x] \exp \left[-i \int_{0}^{s} \mathrm{~d} \sigma\left(\frac{1}{4} g_{\mu \nu} \frac{\mathrm{d} x^{\mu}}{\mathrm{d} \sigma} \frac{\mathrm{d} x^{\nu}}{\mathrm{d} \sigma}+\tilde{\xi} R(x)\right)\right] .
$$

The first claim of such a representation of the massive scalar Green's function $G\left(x, x^{\prime}\right)$ in a gravitational background as a worldline path integral goes back to De Witt [113] and Parker $[114,115] .{ }^{3}$ One issue is that the path integral measure becomes metric dependent, i.e. schematically one has

$$
\mathcal{D}[x]=D[x] \prod_{0 \leq \sigma \leq s} \sqrt{-\operatorname{det} g_{\mu \nu}[x(\sigma)]},
$$

where $D[x]=\prod_{\sigma} \mathrm{d}^{D} x(\sigma)$ is the standard flat space path integral measure. This metric dependence may be conveniently controlled through bosonic $\mathfrak{a}^{\mu}$ and fermionic $\mathfrak{b}^{\mu}, \mathfrak{c}^{\mu}$ "LeeYang" ghosts $[116]:^{4}$

$$
\prod_{0 \leq \sigma \leq s} \sqrt{-\operatorname{det} g_{\mu \nu}[x(\sigma)]}=\int D[\mathfrak{a}, \mathfrak{b}, \mathfrak{c}] \exp \left[-i \int_{0}^{s} \mathrm{~d} \sigma\left(\frac{1}{4} g_{\mu \nu}\left(\mathfrak{a}^{\mu} \mathfrak{a}^{\nu}+\mathfrak{b}^{\mu} \mathfrak{c}^{\nu}\right)\right]\right.
$$

With these ghosts included all divergences in the worldline QFT have been shown to cancel, yet a finite counter term $\frac{1}{4} R(x)$ remains [102]. ${ }^{5}$

\footnotetext{
${ }^{2}$ The derivation of this classic result is nicely reviewed in chapter 33 of ref. [108].

${ }^{3}$ They wrongly claimed this result with $\tilde{\xi}=\xi-\frac{1}{3}$, with $\xi$ the non-minimal coupling of eq. (2.8).

${ }^{4}$ The fermionic path integral yields a factor of $[-\operatorname{det} g]$ while the bosonic one contributes $[-\operatorname{det} g]^{-1 / 2}$ yielding the desired total $[-\operatorname{det} g]^{1 / 2}$.

${ }^{5}$ In non-covariant regularization schemes, such as mode regularization, additional terms proportional to Christoffel symbols appear, $-\frac{1}{12} g^{\mu \nu} g^{\rho \kappa} g_{\sigma \eta} \Gamma_{\mu \rho}^{\sigma} \Gamma_{\nu \kappa}^{\eta}$.
} 


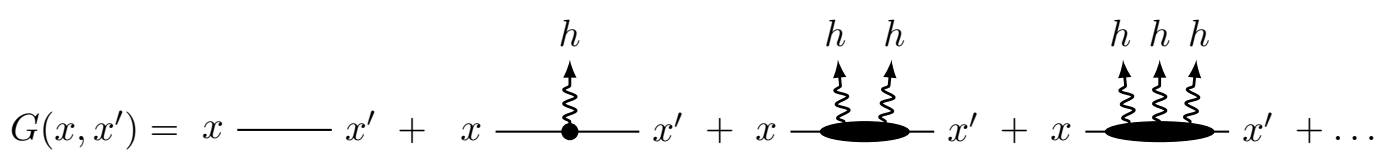

Figure 1. Graphical representation of the Green function $G\left(x, x^{\prime}\right)$ of eq. (2.16) for a massive scalar moving in a weak gravitational background $g_{\mu \nu}=\eta_{\mu \nu}+\kappa h_{\mu \nu}$, excluding the $R \phi^{2}$ interaction in eq. (2.8). A closed expression for the Green's function in momentum space may be found in eq. (3.7).

The upshot is the following representation of the scalar Green's function in a gravitational background that generalizes eq. (2.11) to the gravitational case [102, 103]:

$$
\begin{aligned}
G\left(x, x^{\prime}\right)= & \int_{0}^{\infty} \mathrm{d} s e^{-i s m^{2}} \int_{x(0)=x}^{x(s)=x^{\prime}} D[x] \int D[\mathfrak{a}, \mathfrak{b}, \mathfrak{c}] \\
& \times \times \exp \left[-i \int_{0}^{s} \mathrm{~d} \sigma\left(\frac{1}{4} g_{\mu \nu}\left(\frac{\mathrm{d} x^{\mu}}{\mathrm{d} \sigma} \frac{\mathrm{d} x^{\nu}}{\mathrm{d} \sigma}+\mathfrak{a}^{\mu} \mathfrak{a}^{\nu}+\mathfrak{b}^{\mu} \mathfrak{c}^{\nu}\right)+\left(\xi-\frac{1}{4}\right) R(x)\right)\right] .
\end{aligned}
$$

Writing $\sigma=\frac{\tau}{2 m}$ (where $\tau$ is the proper time) and $s=\frac{T}{2 m}$ yields an expression excitingly close to the worldline action $S_{\mathrm{pm}}$ we obtained in eq. (2.7):

$$
\begin{aligned}
G\left(x, x^{\prime}\right)= & \int_{0}^{\infty} \frac{\mathrm{d} T}{2 m} \int_{x(0)=x}^{x(T)=x^{\prime}} D[x] \exp \left[-i \int_{0}^{T} \mathrm{~d} \tau\left(\frac{m}{2} g_{\mu \nu} \dot{x}^{\mu} \dot{x}^{\nu}+\frac{m}{2}+\frac{1}{2 m}\left(\xi-\frac{1}{4}\right) R(x)\right)\right] \\
& \times \int D[\mathfrak{a}, \mathfrak{b}, \mathfrak{c}] \exp \left[-i \int_{0}^{T} \mathrm{~d} \tau\left(\frac{1}{8 m} g_{\mu \nu}\left(\mathfrak{a}^{\mu} \mathfrak{a}^{\nu}+\mathfrak{b}^{\mu} \mathfrak{c}^{\nu}\right)\right],\right.
\end{aligned}
$$

that is if we ignore the ghosts and the non-minimal coupling to $R$. The ghosts are in fact non-propagating and their purpose in life is to cancel divergences of coinciding worldline fields, i.e. $\left\langle\dot{x}^{\mu}(\tau) \dot{x}^{\nu}(\tau)\right\rangle \sim \delta(0)$. A graphical representation of the gravitationally dressed Green's function in the weak-field approximation is given in figure 1.

\subsection{From the S-matrix to the worldline}

Using the gravitationally dressed Green's function $G$ we can now write S-matrix elements as expectation values of operators in the worldline theory. Assuming a fixed gravitational background we write $G$ as a two-point function via a genuine quantum field theoretical path integral:

$$
G_{i}\left(x, x^{\prime}\right)=\mathcal{Z}_{i}^{-1} \int \mathcal{D}\left[\phi_{i}\right] \phi_{i}(x) \phi_{i}^{\dagger}\left(x^{\prime}\right) e^{i S_{i}}
$$

For the black hole scattering we are interested in we require the S-matrix element of two scalars with or without a final state graviton $\phi_{1} \phi_{2} \rightarrow \phi_{1} \phi_{2}(+h)$ in the classical limit, i.e. suppressing virtual loops in the process. These processes may be computed by inserting two gravitationally dressed Green's functions $G_{i}$ with masses $m_{i}$ into the gravitational path integral. Consider the time-ordered correlator:

$$
\begin{aligned}
\langle\Omega| T\left\{h_{\mu \nu}\right. & \left.(x) \phi_{1}\left(x_{1}\right) \phi_{1}^{\dagger}\left(x_{1}^{\prime}\right) \phi_{2}\left(x_{2}\right) \phi_{2}^{\dagger}\left(x_{2}^{\prime}\right)\right\}|\Omega\rangle \\
& =\tilde{\mathcal{Z}}^{-1} \int \mathcal{D}\left[h_{\mu \nu}, \phi_{1}, \phi_{2}\right] h_{\mu \nu}(x) \phi_{1}\left(x_{1}\right) \phi_{1}^{\dagger}\left(x_{1}^{\prime}\right) \phi_{2}\left(x_{2}\right) \phi_{2}^{\dagger}\left(x_{2}^{\prime}\right) e^{i S^{\prime}} \\
& =\mathcal{Z}^{-1} \int \mathcal{D}\left[h_{\mu \nu}\right] h_{\mu \nu}(x) G_{1}\left(x_{1}, x_{1}^{\prime}\right) G_{2}\left(x_{2}, x_{2}^{\prime}\right) e^{i\left(S_{\mathrm{EH}}+S_{\mathrm{gf}}\right)} .
\end{aligned}
$$


In the last step of integrating out the scalars $\phi_{1}$ and $\phi_{2}$ we have neglected virtual scalar loops that are mediated via gravitons, which is acceptable in the classical limit. For a pure $2 \rightarrow 2$ scattering without a radiated graviton simply drop $h_{\mu \nu}(x)$ above.

The S-matrix then follows via LSZ reduction and Fourier transforming to momentum space:

$$
\begin{aligned}
\left\langle\phi_{1} \phi_{2}(+h)|S| \phi_{1} \phi_{2}\right\rangle= & \mathcal{Z}^{-1} \int \mathrm{d}^{D}\left[x_{i}, x_{i}^{\prime}, x\right] e^{i p_{i} \cdot x_{i}-i p_{i}^{\prime} \cdot x_{i^{\prime}}(-i k \cdot x)} \\
& \times\left.\int \mathcal{D}\left[h_{\mu \nu}\right]\left(\epsilon^{\mu \nu}(k) h_{\mu \nu}(x)\right) G_{1}\left(x_{1}, x_{1}^{\prime}\right) G_{2}\left(x_{2}, x_{2}^{\prime}\right) e^{i\left(S_{\mathrm{EH}}+S_{\mathrm{gf}}\right)}\right|_{\substack{\text { amputated } \\
\text { connected }}} .
\end{aligned}
$$

Note that in the path integral above pure scalar loops never appear, which is why this relation only holds in the $\hbar \rightarrow 0$ limit. The classical limit on the right-hand side then additionally suppresses virtual gravitons in the loops, as well as mixed loops of gravitons and worldline fluctuations that we will describe shortly. Now inserting the worldline path integral representation of the $G_{i}$ from eq. (2.16) on the right-hand side of eq. (2.19) we see that the emerging action in the exponent of the path integral - which should now be interpreted as a QFT on the worldline coupled to the gravitational path integral - is very close to the worldline expression we arrived at in eqs. (2.1) and (2.7). Yet, there are two decisive differences that we shall discuss in turn. Firstly, the worldline action of eq. (2.7) calls for an integral over infinite total proper time $\tau \in[-\infty, \infty]$, whereas in eq. (2.16) we integrate over an ensemble of finite proper times $\tau \in[0, T]$. Secondly, there is the coupling to the Ricci scalar along the worldline appearing in eq. (2.16), which was in principle also allowed in eq. (2.5).

We shall deal with the first point in the following section as it requires a detailed analysis of the LSZ reduction. Addressing the second point, we argue that the non-minimal gravitational $\xi$-coupling of scalars in the action (2.8) is irrelevant for the classical limit of the S-matrix $\phi_{1} \phi_{2} \rightarrow \phi_{1} \phi_{2}(+h)$. For this consider the leading Feynman vertex originating from the interaction term $\xi \int \mathrm{d}^{D} x \sqrt{-g} R \phi_{i}^{\dagger} \phi_{i}$ in eq. (2.8):

$$
\overbrace{\phi_{i}}^{\phi_{i}} \operatorname{mu}_{q} \mu \nu=i \xi \kappa\left(q^{2} \eta_{\mu \nu}-q_{\mu} q_{\nu}\right) \text {. }
$$

The important point is that it couples quadratically to the transfered momentum $q$. As was pointed out in ref. [86] the classical limit of a $\phi_{1} \phi_{2} \rightarrow \phi_{1} \phi_{2}$ scattering process amounts to taking the momentum transfer to zero $(q=\hbar \bar{q}$ with $\hbar \rightarrow 0)$. Hence, there is no contribution of this term to the classical limit of the amplitude. ${ }^{6}$ So we may conveniently set $\xi=\frac{1}{4}$ in eq. (2.16) to remove it from the worldline action. This argument is in line with the arguments presented in ref. [13] for disregarding the Ricci scalar coupling on a worldline quantum field theory in the classical limit.

\footnotetext{
${ }^{6}$ Note that here it is important that $q$ appears quadratically: the linear terms in $q$ in the numerators turn out to be the leading contributions as the $q$-independent ("superclassical") terms cancel out, see [86] and an explicit demonstration in section 5.1.
} 
In summary: we have shown that there is a direct connection between scalar-graviton S-matrices and the worldline QFT in the classical limit via the path integral representation of the gravitationally dressed scalar propagator given in eq. (2.16).

\section{Graviton-dressed propagator for a massive scalar field}

In the previous section we showed how the Feynman-Schwinger representation of a gravitationally dressed scalar propagator could be inserted into a QFT correlator, yielding an expectation value in the worldline theory. However, to study S-matrices we must still apply LSZ reduction. This will convert correlators into S-matrices by cutting the propagators on their external legs, sending those states to the boundary where they interact weakly. In this section we achieve this from the worldline perspective by first deriving a momentum space representation of the gravitationally dressed propagator. The overall effect of putting the scalar legs on-shell is to switch from a worldline action integrated over a finite proper time domain to one over an infinite domain $\tau \in[-\infty, \infty]$. We will then compare with the expectation values one would compute in a worldline QFT. As our first example we examine the eikonal phase of a $2 \rightarrow 2$ S-matrix in the classical limit, which corresponds to the free energy of the worldline theory.

\subsection{Momentum space representation}

Let us now introduce a master formula for the gravitationally dressed two-point function of a massive scalar field coupled to $N$ external gravitons with all legs off-shell, i.e.the momentum space version of $G\left(x, x^{\prime}\right)$ in figure 1 . We work in the non-minimally coupled theory with $\xi=1 / 4$ in eqs. (2.8)-(2.15). To our knowledge only the single-graviton $N=1$ case has been established so far [117].

Starting from the position space propagator $G\left(x, x^{\prime}\right)$ in eq. $(2.15)$ we insert a weak gravitational background of the form

$$
h_{\mu \nu}=\sum_{l=1}^{N} \epsilon_{\mu \nu}^{(l)} e^{i k_{l} \cdot x\left(\sigma_{l}\right)}
$$

into the path integral representing $N$ (off-shell) gravitons - we do not require $k_{l}^{2}=0$ or $k_{l} \cdot \epsilon_{l}=0$. In order to deal with the boundary conditions of the $x^{\mu}(\sigma)$ path integral we perform a background field expansion about straight line trajectories (which solve the flat space equations of motion):

$$
x^{\mu}(\sigma)=x^{\mu}+\Delta x^{\mu} \frac{\sigma}{s}+q^{\mu}(\sigma), \quad \Delta x^{\mu}=x^{\prime \mu}-x^{\mu} .
$$

Inserting this and Fourier transforming eq. (2.15) in $x$ and $x^{\prime}$ to the momentum space variables $p$ and $p^{\prime}$ for the scalar particles yields ${ }^{7}$

$$
\begin{aligned}
D\left(p, p^{\prime},\left\{\epsilon^{(l)}, k_{l}\right\}\right)= & \left(-\frac{i \kappa}{4}\right)^{N} \int_{0}^{\infty} \mathrm{d} s e^{-i s\left(m^{2}-i \epsilon\right)} \int \mathrm{d}^{D} x \int \mathrm{d}^{D} x^{\prime} e^{i\left(p \cdot x-p^{\prime} \cdot x^{\prime}\right)-\frac{i}{4 s} \Delta x^{2}} \\
& \times\left\langle\prod_{l=1}^{N} \int_{0}^{s} \mathrm{~d} \sigma_{l} \epsilon_{\mu \nu}^{(l)}\left(\dot{x}^{\mu}\left(\sigma_{l}\right) \dot{x}^{\nu}\left(\sigma_{l}\right)+\mathfrak{a}^{\mu}\left(\sigma_{l}\right) \mathfrak{a}^{\nu}\left(\sigma_{l}\right)+\mathfrak{b}^{\mu}\left(\sigma_{l}\right) \mathfrak{c}^{\nu}\left(\sigma_{l}\right)\right) e^{i k_{l} \cdot x\left(\sigma_{l}\right)}\right\rangle .
\end{aligned}
$$

\footnotetext{
${ }^{7}$ We include the $i \epsilon$ prescription to make the $s$ integral well-defined. It leads to the bulk Feynman propagator in the final result.
} 
We take $p$ as ingoing and $p^{\prime}$ as outgoing. The expectation value above is defined as an unnormalized path integral over the fluctuations $q$ and the ghost fields:

$$
\langle\mathcal{O}(\mathfrak{a}, \mathfrak{b}, \mathfrak{c}, q)\rangle:=\int D[q, \mathfrak{a}, \mathfrak{b}, \mathfrak{c}] \mathcal{O}(\mathfrak{a}, \mathfrak{b}, \mathfrak{c}, q) e^{-i \int_{0}^{s} \mathrm{~d} \sigma \frac{1}{4}\left(\dot{q}^{2}+\mathfrak{a}^{2}+\mathfrak{b} \cdot \mathfrak{c}\right)} .
$$

All fluctuating fields now have vanishing boundary conditions.

Our task now is to evaluate the correlator in eq. (3.3), and then take the Fourier transform. For this we insert the relevant two-point functions on the worldline:

$$
\begin{aligned}
& \left\langle q^{\mu}(\sigma) q^{\nu}\left(\sigma^{\prime}\right)\right\rangle=2 i \eta^{\mu \nu} \Delta\left(\sigma, \sigma^{\prime}\right), \\
& \left\langle\mathfrak{a}^{\mu}(\sigma) \mathfrak{a}^{\nu}\left(\sigma^{\prime}\right)\right\rangle=-2 i \eta^{\mu \nu} \delta\left(\sigma-\sigma^{\prime}\right), \\
& \left\langle\mathfrak{b}^{\mu}(\sigma) \mathfrak{c}^{\nu}\left(\sigma^{\prime}\right)\right\rangle=4 i \eta^{\mu \nu} \delta\left(\sigma-\sigma^{\prime}\right),
\end{aligned}
$$

where the propagator on a worldline of finite length $s$ is (see e.g.ref. [111])

$$
\Delta\left(\sigma, \sigma^{\prime}\right)=\frac{1}{2}\left|\sigma-\sigma^{\prime}\right|+\frac{\sigma \sigma^{\prime}}{s}-\frac{\sigma+\sigma^{\prime}}{2}, \quad \text { with } \sigma, \sigma^{\prime} \in[0, s] .
$$

It is a straightforward exercise to evaluate the path integrals, though as the details are somewhat involved a full discussion is relegated to appendix A. The final result is a compact master formula for the gravitationally dressed scalar propagator:

$$
\begin{aligned}
D\left(p, p^{\prime},\left\{\epsilon^{(l)}, k_{l}\right\}\right)= & \left(-\frac{i \kappa}{4}\right)^{N} \delta^{(D)}\left(p-p^{\prime}+\sum_{l=1}^{N} k_{l}\right) \int_{0}^{\infty} \mathrm{d} s e^{i s\left(p^{\prime 2}-m^{2}+i \epsilon\right)} \\
& \times \prod_{l=1}^{N} \int_{0}^{s} \mathrm{~d} \sigma_{l} \epsilon^{(l), \mu \nu}\left[\partial_{\epsilon_{l}^{\mu}} \partial_{\epsilon_{l}^{\nu}}+\partial_{\alpha_{l}^{\mu}} \partial_{\alpha_{l}^{\nu}}+\partial_{\beta_{l}^{\mu}} \partial_{\gamma_{l}^{\nu}}\right] \\
& \times \exp \left[-\left(p+p^{\prime}\right) \cdot \sum_{l=1}^{N}\left(i k_{l} \sigma_{l}+\epsilon_{l}\right)\right. \\
& -i \sum_{l, l^{\prime}=1}^{N}\left\{\frac{\left|\sigma_{l}-\sigma_{l^{\prime}}\right|}{2} k_{l} \cdot k_{l^{\prime}}-i \operatorname{sign}\left(\sigma_{l}-\sigma_{l^{\prime}}\right) \epsilon_{l} \cdot k_{l^{\prime}}\right. \\
& \left.\left.+\delta\left(\sigma_{l}-\sigma_{l^{\prime}}\right)\left(\epsilon_{l} \cdot \epsilon_{l^{\prime}}+\alpha_{l} \cdot \alpha_{l^{\prime}}-4 \gamma_{l} \cdot \beta_{l^{\prime}}\right)\right\}\right]\left.\right|_{\epsilon_{l}=\alpha_{l}=\beta_{l}=\gamma_{l}=0}
\end{aligned}
$$

Here we have introduced fiducial "polarization" vectors $\epsilon_{l}^{\mu}$ and $\alpha_{l}^{\mu}$, as well as anticommuting vectors $\beta_{l}^{\mu}$ and $\gamma_{l}^{\mu}$. The expression is remarkably similar (in the double copy sense) to the one obtained for the $N$-photon-dressed $[118,119]$ propagator : to insert a photon leg in lieu of a graviton one simply takes a single $\partial_{\epsilon_{l}^{\mu}}$ derivative there. ${ }^{8}$

To better understand this formula it is instructive to work out the single graviton $(N=$ 1) case. Noting $\operatorname{sign}(0)=0$ and the cancellation of the $\delta(0)$ terms when all polarization derivatives hit the same leg $l=l^{\prime}$, i.e.

$$
\epsilon^{(l), \mu \nu}\left[\partial_{\epsilon_{l}^{\mu}} \partial_{\epsilon_{l}^{\nu}}+\partial_{\alpha_{l}^{\mu}} \partial_{\alpha_{l}^{\nu}}+\partial_{\beta_{l}^{\mu}} \partial_{\gamma_{l}^{\nu}}\right]\left(\epsilon_{l} \cdot \epsilon_{l}+\alpha_{l} \cdot \alpha_{l}-4 \gamma_{l} \cdot \beta_{l}\right)=(2+2-4) \epsilon^{(l), \mu}{ }_{\mu}=0
$$

\footnotetext{
${ }^{8}$ It would be interesting to work out the double copy relation to the $N$-gluon dressed propagator found in $[120]$ in detail.
} 
one straightforwardly works out

$$
D\left(p, p^{\prime}, k ; \epsilon\right)=\frac{i}{p^{2}-m^{2}+i \epsilon} \frac{i}{p^{2}-m^{2}+i \epsilon}\left(-\frac{i \kappa}{4}\right) \delta^{(D)}\left(p-p^{\prime}+k\right)\left(p+p^{\prime}\right)^{\mu}\left(p+p^{\prime}\right)^{\nu} \epsilon_{\mu \nu},
$$

reproducing ref. [117]. Amputating the scalar legs and stripping off the momentumconserving $\delta^{(D)}(P)$ function and polarization tensor we obtain the three-point vertex

$$
-\frac{i \kappa}{4}\left(p+p^{\prime}\right)^{\mu}\left(p+p^{\prime}\right)^{\nu}
$$

Let us compare this result to the QFT three-point vertex of two scalars and a graviton. For a general $\xi$ coupling there are two vertices: the three-point interaction vertex between two scalars $\varphi$ and a graviton $h_{\mu \nu}$ from the minimal coupling (all scalar momenta ingoing):

$$
p \longrightarrow \longrightarrow \vec{\xi}_{q} p^{\prime}=-i \kappa\left[p^{(\mu} p^{\nu)}-\frac{\eta^{\mu \nu}}{2}\left(p \cdot p^{\prime}-m^{2}\right)\right] .
$$

To this we need to add the non-minimal $\xi$ coupling vertex of eq. (2.20)

$$
\begin{aligned}
\int_{p_{q}^{\prime}}^{p_{q}}+\underbrace{p_{q}}_{p^{\prime}} \mu \nu & =-i \kappa\left[p^{(\mu} p^{\prime \nu)}-\frac{\eta^{\mu \nu}}{2}\left(p \cdot p^{\prime}-m^{2}\right)-\xi\left(q^{2} \eta_{\mu \nu}-q_{\mu} q_{\nu}\right)\right] \\
& =-i \kappa\left[\frac{1}{4}\left(p+p^{\prime}\right)^{\mu}\left(p+p^{\prime}\right)^{\nu}+\left(\frac{1}{4}-\xi\right)\left(q^{2} \eta_{\mu \nu}-q_{\mu} q_{\nu}\right)\right]
\end{aligned}
$$

where - crucially - in the last line we have used the on-shell condition $p^{2}=m^{2}=p^{2}$ on the scalar legs. We have a match for $\xi=\frac{1}{4}$, but only if we put the scalar legs on-shell. ${ }^{9}$

It is a simple exercise to also include the $\tilde{\xi} R[x(\tau)]$ term in the worldline action and perform the path integral for $N=1$ as well. One quickly arrives at the above expression for the general $\xi$ case.

\subsection{Putting the scalar legs on shell}

Now that we have a momentum space representation of the gravitationally dressed scalar propagator we can proceed to put the scalar legs on-shell. As we have already seen, this is necessary in order to match to the QFT expression which is then effectively a form factor $F\left(p, p^{\prime} ;\left\{k_{i}, \epsilon_{i}\right\}\right)$ with off-shell graviton legs:

$$
F\left(p, p^{\prime} \mid\left\{k_{i}, \epsilon_{i}\right\}\right)=\left\langle-p^{\prime}\left|\prod_{i=1}^{N} \epsilon_{i} \cdot h\left(k_{i}\right)\right| p\right\rangle=p \rightarrow \sum^{\epsilon_{1}, k_{1}} p^{\prime} .
$$

\footnotetext{
${ }^{9}$ This might be in fact the simplest derivation of the $\tilde{\xi}=\xi-\frac{1}{4}$ relation in eq. (2.15).
} 
Let us perform the LSZ reduction on $D\left(p, p^{\prime},\{\epsilon, k\}\right)$ of eq. (3.7) now. First we put the outgoing $p^{\prime}$ scalar leg on shell:

$$
-\left.i\left(p^{2}-m^{2}+i \epsilon\right) D\left(p, p^{\prime},\{\epsilon, k\}\right)\right|_{p^{\prime 2}=m^{2}-i \epsilon} .
$$

Therefore we pull the inverse propagator into the $s$ integral in eq. (3.7) and use

$$
-i\left(p^{\prime 2}-m^{2}+i \epsilon\right) \int_{0}^{\infty} \mathrm{d} s e^{i s\left(p^{\prime 2}-m^{2}+i \epsilon\right)} \Omega(s)=-\int_{0}^{\infty} \mathrm{d} s \frac{\mathrm{d}}{\mathrm{d} s}\left(e^{i s\left(p^{\prime 2}-m^{2}+i \epsilon\right)}\right) \Omega(s)
$$

where we have introduced

$$
\begin{aligned}
\Omega(s):= & \left(-\frac{i \kappa}{4}\right)^{N} \delta^{(D)}\left(p-p^{\prime}+\sum_{l=1}^{N} k_{l}\right) \prod_{l=1}^{N} \int_{0}^{s} \mathrm{~d} \sigma_{l} \epsilon^{(l), \mu \nu}\left[\partial_{\epsilon_{l}^{\mu}} \partial_{\epsilon_{l}^{\nu}}+\partial_{\alpha_{l}^{\mu}} \partial_{\alpha_{l}^{\nu}}+\partial_{\beta_{l}^{\mu}} \partial_{\gamma_{l}^{\nu}}\right] \\
& \times \exp \left[-\left(p+p^{\prime}\right) \cdot \sum_{l=1}^{N}\left(i k_{l} \sigma_{l}+\epsilon_{l}\right)-i \sum_{l, l^{\prime}=1}^{N}\left\{\frac{\left|\sigma_{l}-\sigma_{l^{\prime}}\right|}{2} k_{l} \cdot k_{l^{\prime}}-i \operatorname{sign}\left(\sigma_{l}-\sigma_{l^{\prime}}\right) \epsilon_{l} \cdot k_{l^{\prime}}\right.\right. \\
& \left.\left.+\delta\left(\sigma_{l}-\sigma_{l^{\prime}}\right)\left(\epsilon_{l} \cdot \epsilon_{l^{\prime}}+\alpha_{l} \cdot \alpha_{l^{\prime}}-4 \gamma_{l} \cdot \beta_{l^{\prime}}\right)\right\}\right]\left.\right|_{\epsilon_{l}=\alpha_{l}=\beta_{l}=\gamma_{l}=0} .
\end{aligned}
$$

Partially integrating eq. (3.14) and using $\Omega(0)=0$ yields

$$
-\left.i\left(p^{\prime 2}-m^{2}+i \epsilon\right) D\left(p, p^{\prime},\{\epsilon, k\}\right)\right|_{p^{\prime 2}=m^{2}-i \epsilon}=\left.\Omega(\infty)\right|_{p^{\prime 2}=m^{2}-i \epsilon} .
$$

The overall effect is therefore to send $s \rightarrow \infty .^{10}$

It remains to put the incoming $p$ scalar leg on shell. For this we first go to "center of mass" proper time coordinates:

$$
\tilde{\sigma}_{l}:=\sigma_{l}-\sigma_{+} \quad \text { with } \quad \sigma_{+}=\frac{1}{N} \sum_{l=1}^{N} \sigma_{l},
$$

and we pick up the constraint $\sum_{l} \tilde{\sigma}_{l}=0$. The $N$-fold integral over the $\sigma_{l}$ 's may then be rewritten as

$$
\prod_{l=1}^{N} \int_{0}^{\infty} \mathrm{d} \sigma_{l} \ldots=\prod_{l=1}^{N} \int_{-\infty}^{\infty} \mathrm{d} \tilde{\sigma}_{l} \int_{0}^{\infty} \mathrm{d} \sigma_{+} \delta\left(\sum_{l=1}^{N} \tilde{\sigma}_{l}\right) \ldots
$$

Note the change of the integration region to $\mathbb{R}$ in the new proper-time coordinates $\tilde{\sigma}_{l}$ matching the one performed in the worldline QFT. Moreover, as $\sigma_{l}-\sigma_{l^{\prime}}=\tilde{\sigma}_{l}-\tilde{\sigma}_{l^{\prime}}$ the variable $\sigma_{+}$only couples to the $\exp \left[-i\left(p+p^{\prime}\right) \cdot \sum_{l} k_{l} \sigma_{l}\right]$ term in $\Omega(\infty)$ from eq. (3.15). One then easily performs the $\sigma_{+}$integral:

$$
\begin{aligned}
\int_{0}^{\infty} \mathrm{d} \sigma_{+} e^{-i\left(p+p^{\prime}\right) \cdot \sum_{l=1}^{N} k_{l} \sigma_{l}} & =e^{-i\left(p+p^{\prime}\right) \cdot \sum_{l=1}^{N} k_{l} \tilde{\sigma}_{l}} \int_{0}^{\infty} \mathrm{d} \sigma_{+} e^{i\left(p+p^{\prime}\right) \cdot\left(p-p^{\prime}\right) \sigma_{+}} \\
& =\frac{i e^{-i\left(p+p^{\prime}\right) \cdot \sum_{l=1}^{N} k_{l} \tilde{\sigma}_{l}}}{p^{2}-m^{2}+i \epsilon}
\end{aligned}
$$

\footnotetext{
${ }^{10}$ See also the recent [121] making the same argument.
} 
where we have used total momentum conservation and the mass-shell condition for $p^{\prime}$. But this precisely extracts the incoming scalar propagator!

Hence the net effect of LSZ reducing the graviton dressed propagator of eq. (3.7) to a form factor is very mild and can be done explicitly: drop the overall $s$ integral, insert a total proper-time delta function and take the proper time integrals to run over $\mathbb{R} .{ }^{11}$ The final result is (dropping the tildes on $\sigma$ )

$$
\begin{aligned}
F\left(p, p^{\prime} \mid\left\{\epsilon^{(l)}, k_{l}\right\}\right)= & \left(-\frac{i \kappa}{4}\right)^{N} \delta^{(D)}(P) \prod_{l=1}^{N}\left[\int_{-\infty}^{\infty} \mathrm{d} \sigma_{l} \epsilon^{(l), \mu \nu}\left(\partial_{\epsilon_{l}^{\mu}} \partial_{\epsilon_{l}^{\nu}}+\partial_{\alpha_{l}^{\mu}} \partial_{\alpha_{l}^{\nu}}+\partial_{\beta_{l}^{\mu}} \partial_{\gamma_{l}^{\nu}}\right)\right] \\
& \times \delta\left(\sum_{l=1}^{N} \sigma_{l}\right) \exp \left[-\left(p+p^{\prime}\right) \cdot \sum_{l=1}^{N}\left(i k_{l} \sigma_{l}+\epsilon_{l}\right)\right. \\
& -i \sum_{l, l^{\prime}=1}^{N}\left\{\frac{\left|\sigma_{l}-\sigma_{l^{\prime}}\right|}{2} k_{l} \cdot k_{l^{\prime}}-i \operatorname{sign}\left(\sigma_{l}-\sigma_{l^{\prime}}\right) \epsilon_{l} \cdot k_{l^{\prime}}\right. \\
& \left.\left.+\delta\left(\sigma_{l}-\sigma_{l^{\prime}}\right)\left(\epsilon_{l} \cdot \epsilon_{l^{\prime}}+\alpha_{l} \cdot \alpha_{l^{\prime}}-4 \gamma_{l} \cdot \beta_{l^{\prime}}\right)\right\}\right]\left.\right|_{\substack{\epsilon_{l}=\alpha_{l}=\beta_{l}=\gamma_{l}=0 \\
p^{2}=m^{2}-i \epsilon=p^{\prime 2}}}
\end{aligned}
$$

with $P=p-p^{\prime}+\sum_{l} k_{l}$. This is a surprisingly compact result for an $N$-graviton emission expression.

\subsection{Link to position space expression}

Let us see how this form factor relates to the analogous expressions one would compute in a worldline QFT (WQFT). Here the starting point is that of eq. (2.15), except with an integral over infinitely extended proper times:

$$
\Xi\left(b, v ;\left\{\epsilon^{(l)}, k_{l}\right\}\right):=\int D[x] \int D[\mathfrak{a}, \mathfrak{b}, \mathfrak{c}] \exp \left[-i \int_{-\infty}^{\infty} \mathrm{d} \sigma\left(\frac{1}{4} g_{\mu \nu}\left(\dot{x}^{\mu} \dot{x}^{\nu}+\mathfrak{a}^{\mu} \mathfrak{a}^{\nu}+\mathfrak{b}^{\mu} \mathfrak{c}^{\nu}\right)\right)\right],
$$

where again we begin with a collection of plane waves for the graviton with momenta $k_{l}$ and polarizations $\epsilon^{(l)}$ : eq. (3.1). This is equivalent to

$$
\begin{aligned}
\Xi(b, v & \left.;\left\{\epsilon^{(l)}, k_{l}\right\}\right) \\
= & \left(-\frac{i \kappa}{4}\right)^{N} \int D[x] \int D[\mathfrak{a}, \mathfrak{b}, \mathfrak{c}] \prod_{l=1}^{\infty}\left[\int _ { - \infty } ^ { \infty } d \sigma _ { l } \epsilon _ { l } ^ { \mu \nu } \left(\dot{x}^{\mu}\left(\sigma_{l}\right) \dot{x}^{\nu}\left(\sigma_{l}\right)+\mathfrak{a}^{\mu}\left(\sigma_{l}\right) \mathfrak{a}^{\nu}\left(\sigma_{l}\right)\right.\right. \\
& \left.\left.+\mathfrak{b}^{\mu}\left(\sigma_{l}\right) \mathfrak{c}^{\nu}\left(\sigma_{l}\right)\right) e^{i k_{l} \cdot x\left(\sigma_{l}\right)}\right] \exp \left[-i \int_{-\infty}^{\infty} \mathrm{d} \sigma\left(\frac{1}{4}\left(\dot{x}^{2}(\sigma)+\mathfrak{a}^{2}(\sigma)+\mathfrak{b}(\sigma) \cdot \mathfrak{c}(\sigma)\right)\right)\right] .
\end{aligned}
$$

We note from the action appearing in the last exponential that the momentum associated to $x^{\mu}$ is $p_{\mu}=-\frac{1}{2} \dot{x}_{\mu}$, which is somewhat unconventional. Inserting the proper time $\tau=2 m \sigma$ as done above eq. (2.16) would yield the canonical relation.

\footnotetext{
${ }^{11}$ These steps to put the scalar legs on shell apply generically to any Feynman-Schwinger representation of a gluon, photon or graviton dressed propagator, and have to the best of our knowledge not been observed before.
} 
We now consider the background field expansion for $x^{\mu}(\sigma)$ :

$$
x^{\mu}(\sigma)=b^{\mu}+v^{\mu} \sigma+z^{\mu}(\sigma) .
$$

In order to integrate out the $z^{\mu}$ field and the ghosts we use generic translation-invariant propagators:

$$
\begin{aligned}
& \left\langle z^{\mu}(\sigma) z^{\nu}\left(\sigma^{\prime}\right)\right\rangle=2 i \eta^{\mu \nu} \Delta\left(\sigma-\sigma^{\prime}\right), \\
& \left\langle\mathfrak{a}^{\mu}(\sigma) \mathfrak{a}^{\nu}\left(\sigma^{\prime}\right)\right\rangle=-2 i \eta^{\mu \nu} \delta\left(\sigma-\sigma^{\prime}\right), \\
& \left\langle\mathfrak{b}^{\mu}(\sigma) \mathfrak{c}^{\nu}\left(\sigma^{\prime}\right)\right\rangle=+4 i \eta^{\mu \nu} \delta\left(\sigma-\sigma^{\prime}\right) .
\end{aligned}
$$

Concerning $\Delta(\sigma)$ we shall at this point only assume that $\partial_{\sigma} \partial_{\sigma^{\prime}} \Delta\left(\sigma-\sigma^{\prime}\right)=-\delta\left(\sigma-\sigma^{\prime}\right)$, which holds true for a time-symmetric as well as retarded (or advanced) propagator on the infinitely extended worldline. With this one straightforwardly finds (again going to "center of mass" proper time coordinates as we did in eq. (3.17)):

$$
\begin{aligned}
\Xi\left(b, v ;\left\{\epsilon^{(l)}, k_{l}\right\}\right)= & \Xi_{0} \delta\left(\sum_{l=1}^{N} k_{l} \cdot v\right) e^{i \sum_{l=1}^{N} k_{l} \cdot b} \\
& \times\left(-\frac{i \kappa}{4}\right)^{N} \prod_{l=1}^{N}\left[\int_{-\infty}^{\infty} \mathrm{d} \sigma_{l} \epsilon^{(l), \mu \nu}\left(\partial_{\epsilon_{l}^{\mu}} \partial_{\epsilon_{l}^{\nu}}+\partial_{\alpha_{l}^{\mu}} \partial_{\alpha_{l}^{\nu}}+\partial_{\beta_{l}^{\mu}} \partial_{\gamma_{l}^{\nu}}\right)\right] \\
& \times \delta\left(\sum_{l=1}^{N} \sigma_{l}\right) \exp \left[v \cdot \sum_{l=1}^{N}\left(i k_{l} \sigma_{l}+\epsilon_{l}\right)\right. \\
& -i \sum_{l, l^{\prime}=1}^{N}\left\{\Delta\left(\sigma_{l}-\sigma_{l^{\prime}}\right) k_{l} \cdot k_{l^{\prime}}-i \partial_{\sigma_{l}} \Delta\left(\sigma_{l}-\sigma_{l^{\prime}}\right) \epsilon_{l} \cdot k_{l^{\prime}}\right. \\
& \left.\left.+\delta\left(\sigma_{l}-\sigma_{l^{\prime}}\right)\left(\epsilon_{l} \cdot \epsilon_{l^{\prime}}+\alpha_{l} \cdot \alpha_{l^{\prime}}-4 \gamma_{l} \cdot \beta_{l^{\prime}}\right)\right\}\right]\left.\right|_{\epsilon_{l}=\alpha_{l}=\beta_{l}=\gamma_{l}=0}
\end{aligned}
$$

Here $\Xi_{0}$ is an overall measure factor

$$
\Xi_{0}:=\lim _{T \rightarrow \infty}\left[\frac{i}{(2 \pi T)^{d / 2}} e^{-i T v^{2} / 2}\right]
$$

that we may drop as it falls out of normalized correlation functions. Now if we identify the boundary conditions in terms of the momenta as (recall $p_{\mu}=-\frac{1}{2} \frac{d x_{\mu}}{d \sigma}$ )

$$
p^{\mu}=-\frac{1}{2} \dot{x}(-\infty)=-\frac{v^{\mu}}{2}+\frac{q^{\mu}}{2}, \quad p^{\prime \mu}=-\frac{1}{2} \dot{x}(+\infty)=-\frac{v^{\mu}}{2}-\frac{q^{\mu}}{2},
$$

where $q$ is the total momentum transfer of the scattered scalar particle, we see that (3.25) is dauntingly close to the form factor expression (3.20) upon noting that $-v=p+p^{\prime}$ ! Concretely, if we pick the worldline propagator to be time-symmetric,

$$
\Delta(\sigma)=\frac{|\sigma|}{2}
$$

we arrive at our central relation linking the QFT form factor to the WQFT correlator:

$$
\frac{\Xi\left(b, v ;\left\{\epsilon^{(l)}, k_{l}\right\}\right)}{\Xi_{0}}=\delta\left(\sum_{l=1}^{N} k_{l} \cdot v\right) e^{i \sum_{l=1}^{N} k_{l} \cdot b} F\left(p, p^{\prime} \mid\left\{\epsilon^{(l)}, k_{l}\right\}\right),
$$


where the use of Feynman propagators is understood in the form factor. Note the emergence of the total momentum transfer $q=\sum_{l=1}^{N} k_{l}$ in the above.

So that the significance of eq. (3.29) is properly understood, let us briefly recap the steps that have led us here. We started with the scalar Green's function $G\left(x, x^{\prime}\right)$ in a gravitational background (2.15), which can be inserted into time-ordered correlation functions containing pairs of distinctly flavored scalars - see eq. (2.18). Moving from time-ordered correlators to S-matrices required us to obtain a momentum space representation of $G\left(x, x^{\prime}\right)$ - $D\left(p, p^{\prime},\left\{\epsilon^{(l)}, k_{l}\right\}\right)$, given in eq. (3.7) - and then cut into its external scalar legs, yielding the form factor $F\left(p, p^{\prime} \mid\left\{\epsilon^{(l)}, k_{l}\right\}\right)$. What eq. (3.29) therefore tells us - provided the external legs are on shell - is that we can identify S-matrices with expectation values in the WQFT using the classical $\hbar \rightarrow 0$ limit. The expectation values in the $n$-body case are ${ }^{12}$

$$
\begin{aligned}
\left\langle\mathcal{O}\left(h,\left\{x_{i}\right\}\right)\right\rangle_{\mathrm{WQFT}}= & \mathcal{Z}_{\mathrm{WQFT}}^{-1} \int D\left[h_{\mu \nu}\right] \int \prod_{i=1}^{n} D\left[z_{i}, \mathfrak{a}_{i}, \mathfrak{b}_{i}, \mathfrak{c}_{i}\right] \mathcal{O}\left(h,\left\{x_{i}\right\}\right) e^{i\left(S_{\mathrm{EH}}+S_{\mathrm{gf}}\right)} \\
& \times \exp \left[-i \sum_{i=1}^{n} \int_{-\infty}^{\infty} \mathrm{d} \tau_{i} \frac{m_{i}}{2} g_{\mu \nu}\left(\dot{x}_{i}^{\mu} \dot{x}_{i}^{\nu}+\mathfrak{a}_{i}^{\mu} \mathfrak{a}_{i}^{\nu}+\mathfrak{b}_{i}^{\mu} \mathfrak{c}_{i}^{\nu}\right)\right]
\end{aligned}
$$

where $g_{\mu \nu}(x)=\eta_{\mu \nu}+\kappa h_{\mu \nu}(x)$ and $x_{i}\left(\tau_{i}\right)=b_{i}+v_{i} \tau_{i}+z_{i}\left(\tau_{i}\right)$. $\mathcal{Z}_{\mathrm{WQFT}}$ is the partition function

$$
\begin{aligned}
\mathcal{Z}_{\mathrm{WQFT}}:= & \text { const } \times \int D\left[h_{\mu \nu}\right] \int \prod_{i=1}^{n} D\left[z_{i}, \mathfrak{a}_{i}, \mathfrak{b}_{i}, \mathfrak{c}_{i}\right] e^{i\left(S_{\mathrm{EH}}+S_{\mathrm{gf}}\right)} \\
& \times \exp \left[-i \sum_{i=1}^{n} \int_{-\infty}^{\infty} \mathrm{d} \tau_{i} \frac{m_{i}}{2} g_{\mu \nu}\left(\dot{x}_{i}^{\mu} \dot{x}_{i}^{\nu}+\mathfrak{a}_{i}^{\mu} \mathfrak{a}_{i}^{\nu}+\mathfrak{b}_{i}^{\mu} \mathfrak{c}_{i}^{\nu}\right)\right],
\end{aligned}
$$

and const ensures that $\mathcal{Z}_{\mathrm{WQFT}}=1$ in the non-interacting case $(\kappa=0)$.

\subsection{Towards the eikonal phase}

Equipped with eq. (3.29) we discover an intriguing relation between the free energy of the WQFT and the eikonal phase of a $2 \rightarrow 2$ scalar S-matrix in the classical limit. The exponentiated eikonal phase is defined as a Fourier transform of the S-matrix into impact parameter space transverse to the $(D-2)$-dimensional scattering plane $[106,122]$ :

$$
e^{i \chi}:=\frac{1}{4 m_{1} m_{2}} \int \frac{\mathrm{d}^{D} q}{(2 \pi)^{D-2}} \delta\left(q \cdot v_{1}\right) \delta\left(q \cdot v_{2}\right) e^{i q \cdot b}\left\langle\phi_{1} \phi_{2}|S| \phi_{1} \phi_{2}\right\rangle
$$

where $b=b_{2}-b_{1}$ and $q=p_{1}^{\prime}-p_{1}=p_{2}-p_{2}^{\prime}$ is the momentum transfer from particle 1 to 2 ( $p_{i}$ momenta ingoing and $p_{i}^{\prime}$ momenta outgoing).

An immediate corollary of eq. (2.19) and eq. (3.29) and a central result of our work is then the simple relation (holding in the classical limit)

$$
\mathcal{Z}_{\mathrm{WQFT}}=e^{i \chi}
$$

\footnotetext{
${ }^{12}$ Factors of $\Xi_{0}$ are absorbed into the path integral measure $D\left[z_{i}\right]$.
} 
i.e. the free energy of the WQFT is to be identified with the eikonal phase. This is a rather direct link between the worldline theory and the QFT S-matrix. ${ }^{13}$ We shall evaluate the eikonal phase to $2 \mathrm{PM}$ in section 7 and establish a relationship to the classical impulse $\Delta p_{i}^{\mu}=q^{\mu}$ and scattering angle $\theta$.

\subsection{Time-symmetric vs. retarded propagators}

Let us finally comment on the use of time-symmetric propagators above versus retarded (or advanced) ones in the WQFT. The retarded (or advanced) worldline propagators on an infinite worldline read

$$
\Delta_{r / a}(\sigma)=\frac{|\sigma|}{2} \pm \frac{\sigma}{2}
$$

We claim that switching between these propagators simply amounts to performing shifts in the background parameters $b^{\mu}$ and $v^{\mu}$. This is best seen in a classical setting where one seeks to solve an inhomogeneous second-order ordinary differential equation for $x^{\mu}(\sigma)$. Writing the solution as $x^{\mu}(\sigma)=b^{\mu}+v^{\mu} \sigma+z^{\mu}(\sigma)$, the $b^{\mu}$ and $v^{\mu}$ terms represent a solution to the homogeneous (force-free) equation, whereas the perturbatively constructed $z^{\mu}$ is a specific solution to the inhomogeneous solution.

The choice of propagator is equivalent to picking a specific inhomogeneous solution. Hence all choices for worldline propagators are valid and physically equivalent, but the meaning of the background constants changes. To emphasize this we will denote them as follows: for a retarded propagator $b^{\mu}$ and $v^{\mu}$ describe the initial worldline trajectory $(\sigma \rightarrow-\infty)$, for an advanced propagator $b^{\prime \mu}$ and $v^{\prime \mu}$ give the final worldline $(\sigma \rightarrow+\infty)$, and for a time-symmetric propagator $\hat{b}^{\mu}$ and $\hat{v}^{\mu}$ an in-between state $(\sigma=0)$. So we identify $p^{\mu}=m v^{\mu}, p^{\prime \mu}=m v^{\prime \mu}$ and $\hat{p}^{\mu}=\frac{1}{2}\left(p^{\mu}+p^{\prime \mu}\right)=\hat{m} \hat{v}^{\mu}$ as the ingoing, outgoing, and average momenta respectively, where $\hat{m}^{2}=\frac{m^{2}}{2}\left(1+v \cdot v^{\prime}\right)$ is chosen to ensure $\hat{v}^{2}=1$. One may directly compute the shifts in $b^{\mu}$ and $v^{\mu}$ for transitions between the propagators from their definitions in eqs.(3.28)-(3.34).

The choice of Feynman vs. retarded propagators is also meaningful for the gravitons, but in a different way. The real part of Feynman propagators (as one uses when calculating scattering amplitudes) yield time symmetric Green's functions, which is consistent with purely conservative scattering. For a classically radiating system one should instead use retarded propagators. This will affect observables like the impulse $\Delta p_{i}^{\mu}$, which after integration will have a different form. This important subtlety was recently discussed in the context of the 3PM deflection in ref. [123], resolving a tension with the high-energy limit [90, 98]. It was argued earlier that, from an amplitudes perspective, this tension would be resolved by including the full soft region [124].

\section{WQFT Feynman rules}

In the previous section we saw a clear link between gravitational S-matrices and expectation values of operators evaluated in the WQFT. These involve path integrals over not only the

\footnotetext{
${ }^{13}$ Note that there is a factor $\frac{1}{2 m_{i}}$ for each worldline, which comes from inserting $\sigma_{i}=\frac{1}{2 m_{i}} \tau_{i}$ as described above.
} 
gravitational field $h_{\mu \nu}$, but also the deflection $z^{\mu}$ and ghosts $\mathfrak{a}^{\mu}, \mathfrak{b}^{\mu}, \mathfrak{c}^{\mu}$. In this section we develop a set of Feynman rules which allow us to calculate these expectation values directly. By taking a diagrammatic approach we invite comparisons with the diagrams used to describe scattering amplitudes.

We treat the gravitational field $h_{\mu \nu}(x)$ and deflection $z^{\mu}(\tau)$ on an equal footing. As we are not interested in quantum corrections we will work at tree level, so we can ignore the ghosts. The graviton is most naturally described in momentum space; the deflection in energy space (or frequency, using $E=\hbar \omega$ ):

$$
h_{\mu \nu}(x)=\int_{k} e^{-i k \cdot x} h_{\mu \nu}(k), \quad \quad z^{\mu}(\tau)=\int_{\omega} e^{-i \omega \tau} z^{\mu}(\omega),
$$

where we have introduced the shorthands

$$
\int_{k}:=\int \frac{\mathrm{d}^{4} k}{(2 \pi)^{4}}, \quad \int_{\omega}:=\int \frac{\mathrm{d} \omega}{2 \pi} .
$$

From this point onwards we specialize to $D=4$. We will also absorb factors of $(2 \pi)$ into the $\delta$-functions:

$$
\delta(k):=(2 \pi)^{4} \delta^{(4)}(k), \quad \delta(\omega):=(2 \pi) \delta(\omega) .
$$

The Einstein-Hilbert action (2.2) being integrated over all positions $x$ implies the usual momentum conservation at those interaction vertices; vertices arising from $S_{\mathrm{pm}}$ in (2.7) instead conserve the energy $\omega$.

First consider the Einstein-Hilbert action. The Feynman rules arising from here are the usual ones involving only the graviton $h_{\mu \nu}$, with propagator

$$
h_{\mu \nu}(x) \cos _{k} h_{\rho \sigma}(y)=i P_{\mu \nu ; \rho \sigma} \int_{k} \frac{e^{-i k \cdot(x-y)}}{k^{2}}
$$

where $P_{\mu \nu ; \rho \sigma}=\eta_{\mu(\rho} \eta_{\sigma) \nu}-\frac{1}{2} \eta_{\mu \nu} \eta_{\rho \sigma}$. We are flexible about the $i \epsilon$ prescription: either write the denominator as $k^{2}+i \epsilon$, making it a time-symmetric Feynman propagator, or $\left(k^{0} \pm i \epsilon\right)^{2}-\mathbf{k}^{2}$, making it retarded/advanced. In the retarded case the poles in $k^{0}$ occur at $k^{0}= \pm \sqrt{\mathbf{k}^{2}}-i \epsilon$ : as both are below the real axis the integration contour must be closed in the lower-half plane. So the integral is non-zero only when $x^{0}>y^{0}$, thus ensuring causality.

Next we consider the worldline action $S_{\mathrm{pm}}$ given in eq. (2.7):

$$
S_{\mathrm{pm}}=-\frac{m}{2} \int_{-\infty}^{\infty} \mathrm{d} \tau\left(g_{\mu \nu} \dot{x}^{\mu} \dot{x}^{\nu}+1\right) \text {. }
$$

For now ignoring the parts containing $h_{\mu \nu}$, we expand $\dot{x}^{\mu}(\tau)=v^{\mu}+\dot{z}^{\mu}(\tau)$ to obtain

$$
\left.S_{\mathrm{pm}}\right|_{h_{\mu \nu}=0}=-\int_{-\infty}^{\infty} \mathrm{d} \tau\left(m+m \eta_{\mu \nu} v^{\mu} \dot{z}^{\nu}+\frac{m}{2} \eta_{\mu \nu} \dot{z}^{\mu} \dot{z}^{\nu}\right)
$$

having used $\eta_{\mu \nu} v^{\mu} v^{\nu}=1$. Both the first term (a constant) and the second term (a boundary term) we can ignore; the third gives us our propagator for $z^{\mu}$ :

$$
z^{\mu}\left(\tau_{1}\right) \quad z^{\nu}\left(\tau_{2}\right)=-i \frac{\eta^{\mu \nu}}{m} \int_{\omega} \frac{e^{-i \omega\left(\tau_{1}-\tau_{2}\right)}}{(\omega \pm i \epsilon)^{2}}=\frac{i \eta^{\mu \nu}}{2 m}\left(\left|\tau_{1}-\tau_{2}\right| \pm\left(\tau_{1}-\tau_{2}\right)\right) .
$$


These are the retarded/advanced versions of the propagator, which are non-zero when $\tau_{1}>$ $\tau_{2}$ or $\tau_{1}<\tau_{2}$ respectively. Using $\sigma=\frac{\tau}{2 m}$ we see a precise match for the same propagator given earlier (3.24). We define the time-symmetric propagator as simply the averaged combination of the retarded/advanced propagators. ${ }^{14}$ As we explained in section 3.4, the correct interpretation of $b^{\mu}$ and $v^{\mu}$ is sensitive to the choice of worldline propagator.

Finally we proceed to consider worldline interactions, all of which involve the gravitational field $h_{\mu \nu}$. As $S_{\mathrm{pm}}$ depends on the gravitational field only through $g_{\mu \nu}=\eta_{\mu \nu}+m_{\mathrm{Pl}}^{-1} h_{\mu \nu}$ (and not the inverse metric $g^{\mu \nu}$ ) this conveniently ensures that all such vertices are linear in $h_{\mu \nu}$. We extract the $\tau$ dependence from $h_{\mu \nu}$ when it is evaluated on the worldline of a black hole:

$$
\begin{aligned}
h_{\mu \nu}(x(\tau)) & =\int_{k} e^{i k \cdot(b+v \tau+z(\tau))} h_{\mu \nu}(-k)=\sum_{n=0}^{\infty} \frac{i^{n}}{n !} \int_{k} e^{i k \cdot(b+v \tau)}(k \cdot z(\tau))^{n} h_{\mu \nu}(-k) \\
& =\sum_{n=0}^{\infty} \frac{i^{n}}{n !} \int_{k, \omega_{1}, \ldots, \omega_{n}} e^{i k \cdot b} e^{i\left(k \cdot v+\sum_{i=1}^{n} \omega_{i}\right) \tau}\left(\prod_{i=1}^{n} k \cdot z\left(-\omega_{i}\right)\right) h_{\mu \nu}(-k) .
\end{aligned}
$$

The product on $z^{\mu}\left(-\omega_{i}\right)$ produces a tower of vertices which are fed into the interacting part of the action $S_{\mathrm{pm}}^{\mathrm{int}}=S_{\mathrm{pm}}-\left.S_{\mathrm{pm}}\right|_{h_{\mu \nu}=0}$ :

$$
\begin{aligned}
S_{\mathrm{pm}}^{\mathrm{int}} & =-\frac{m}{2 m_{\mathrm{Pl}}} \int_{-\infty}^{\infty} \mathrm{d} \tau h_{\mu \nu}(x(\tau)) \dot{x}^{\mu}(\tau) \dot{x}^{\nu}(\tau) \\
& =-\frac{m}{2 m_{\mathrm{Pl}}} \int_{-\infty}^{\infty} \mathrm{d} \tau h_{\mu \nu}(x(\tau))\left(v^{\mu} v^{\nu}+2 v^{(\mu} \dot{z}^{\nu)}(\tau)+\dot{z}^{\mu}(\tau) \dot{z}^{\nu}(\tau)\right) .
\end{aligned}
$$

We obtain

$$
\begin{aligned}
S_{\mathrm{pm}}^{\mathrm{int}}= & -\frac{m}{m_{\mathrm{Pl}}} \sum_{n=0}^{\infty} \frac{i^{n}}{n !} \int_{k, \omega_{1}, \ldots, \omega_{n}} e^{i k \cdot b} \delta\left(k \cdot v+\sum_{i=1}^{n} \omega_{i}\right) h_{\mu \nu}(-k)\left(\prod_{i=1}^{n} z^{\rho_{i}}\left(-\omega_{i}\right)\right) \\
& \times\left(\frac{1}{2}\left(\prod_{i=1}^{n} k_{\rho_{i}}\right) v^{\mu} v^{\nu}+\sum_{i=1}^{n} \omega_{i}\left(\prod_{j \neq i}^{n} k_{\rho_{j}}\right) v^{(\mu} \delta_{\rho_{i}}^{\nu)}+\sum_{i<j}^{n} \omega_{i} \omega_{j}\left(\prod_{l \neq i, j}^{n} k_{\rho_{l}}\right) \delta_{\rho_{i}}^{(\mu} \delta_{\rho_{j}}^{\nu)}\right)
\end{aligned}
$$

having integrated over $\tau$ to extract the energy-conserving $\delta$-function. When $n=0$ only the first term in the second line is included; when $n=1$ only the first two terms.

Let us see how the Feynman rules are read off using some explicit examples. At zeroth order in $z^{\mu}$ :

$$
\left.S_{\mathrm{pm}}^{\mathrm{int}}\right|_{z^{0}}=-\frac{m}{2 m_{\mathrm{Pl}}} \int_{k} e^{i k \cdot b} \delta(k \cdot v) h_{\mu \nu}(-k) v^{\mu} v^{\nu} .
$$

This term gives rise to the stress-energy tensor $T^{\mu \nu}(k)=m e^{i k \cdot b} \delta(k \cdot v) v^{\mu} v^{\nu}$ (see e.g.ref. [93]) which we interpret as a classical source for $h_{\mu \nu}$. The Feynman rule is

$$
\sum_{h_{\mu \nu}(k)}=-i \frac{m}{2 m_{\mathrm{Pl}}} e^{i k \cdot b} \delta(k \cdot v) v^{\mu} v^{\nu},
$$

\footnotetext{
${ }^{14}$ For the graviton such an averaging procedure would produce only the real part of its Feynman propagator. The missing imaginary part corresponds to dissipation; however, on the worldline there is no dissipation and hence no imaginary part.
} 
with $k$ outgoing. It is a tadpole: the dotted line represents the worldline, and is intended only as a visual aid. The linear terms in $z^{\mu}$ are

$$
\left.S_{\mathrm{pm}}^{\mathrm{int}}\right|_{z}=-i \frac{m}{2 m_{\mathrm{Pl}}} \int_{k, \omega} e^{i k \cdot b} \delta(k \cdot v+\omega) h_{\mu \nu}(-k) z^{\rho}(-\omega)\left(2 \omega v^{(\mu} \delta_{\rho}^{\nu)}+v^{\mu} v^{\nu} k_{\rho}\right),
$$

from which we read off the two-point vertex:

$$
\sum_{\mu \nu} z^{\rho}(\omega)=\frac{m}{2 m_{\mathrm{Pl}}} e^{i k \cdot b} \delta(k \cdot v+\omega)\left(2 \omega v^{(\mu} \delta_{\rho}^{\nu)}+v^{\mu} v^{\nu} k_{\rho}\right) \text {. }
$$

The energy $\omega$ is also taken as outgoing. Finally, to quadratic order in $z^{\mu}$ :

$$
\begin{aligned}
\left.S_{\mathrm{pm}}^{\mathrm{int}}\right|_{z^{2}}= & \frac{m}{2 m_{\mathrm{Pl}}} \int_{k, \omega_{1}, \omega_{2}} e^{i k \cdot b} \delta\left(k \cdot v+\omega_{1}+\omega_{2}\right) h_{\mu \nu}(-k) z^{\rho_{1}}\left(-\omega_{1}\right) z^{\rho_{2}}\left(-\omega_{2}\right) \\
& \times\left(\frac{1}{2} k_{\rho_{1}} k_{\rho_{2}} v^{\mu} v^{\nu}+\omega_{1} k_{\rho_{2}} v^{(\mu} \delta_{\rho_{1}}^{\nu)}+\omega_{2} k_{\rho_{1}} v^{(\mu} \delta_{\rho_{2}}^{\nu)}+\omega_{1} \omega_{2} \delta_{\rho_{1}}^{(\mu} \delta_{\rho_{2}}^{\nu)}\right) .
\end{aligned}
$$

The associated trivalent Feynman vertex is

$$
\begin{aligned}
& z_{z^{\rho_{2}}\left(\omega_{2}\right)}^{\left.\omega_{1}\right)}= \\
& \sum_{\mu \nu}(k) \frac{m}{m_{\mathrm{Pl}}} e^{i k \cdot b} \delta\left(k \cdot v+\omega_{1}+\omega_{2}\right) \\
& \times\left(\frac{1}{2} k_{\rho_{1}} k_{\rho_{2}} v^{\mu} v^{\nu}+\omega_{1} k_{\rho_{2}} v^{(\mu} \delta_{\rho_{1}}^{\nu)}+\omega_{2} k_{\rho_{1}} v^{(\mu} \delta_{\rho_{2}}^{\nu}+\omega_{1} \omega_{2} \delta_{\rho_{1}}^{(\mu} \delta_{\rho_{2}}^{\nu)}\right) .
\end{aligned}
$$

While of course the second worldline fluctuation still travels on the worldline, we draw it above to distinguish it from its partner.

Given that an $n$-graviton vertex carries an overall $m_{\mathrm{Pl}}^{2-n}$, it might seem odd that each of these $z$-vertices carries only a single power of $m_{\mathrm{Pl}}^{-1}$. To rectify this we might try rescaling $z^{\mu} \rightarrow m_{\mathrm{Pl}}^{-1} z^{\mu}$, similar to how we write $g_{\mu \nu}=\eta_{\mu \nu}+m_{\mathrm{Pl}}^{-1} h_{\mu \nu}$ for the graviton. However, we find this operation to be undesirable as it also rescales the propagator (4.7) to carry an overall $m_{\mathrm{Pl}}^{2}$. As we shall see, despite the higher-point vertices carrying the same overall power of $m_{\mathrm{Pl}}$, their appearance at low orders in the PM expansion is ruled out by the combinatorics of which diagrams we can draw.

The three vertices given above will be sufficient for all of the calculations done in this paper. However, using eq. (4.10) we can easily generalize to an $n$th order vertex:

$$
\begin{aligned}
& V_{\rho_{1} \cdots \rho_{n}}^{\mathrm{WL}, \mu \nu}\left(k ; \omega_{1}, \cdots, \omega_{n}\right) \\
& =i^{n-1} \frac{m}{m_{\mathrm{Pl}}} e^{i k \cdot b} \delta\left(k \cdot v+\sum_{i=1}^{n} \omega_{i}\right) \\
& \quad \times\left(\frac{1}{2}\left(\prod_{i=1}^{n} k_{\rho_{i}}\right) v^{\mu} v^{\nu}+\sum_{i=1}^{n} \omega_{i}\left(\prod_{j \neq i}^{n} k_{\rho_{j}}\right) v^{(\mu} \delta_{\rho_{i}}^{\nu)}+\sum_{i<j}^{n} \omega_{i} \omega_{j}\left(\prod_{l \neq i, j}^{n} k_{\rho_{l}}\right) \delta_{\rho_{i}}^{(\mu} \delta_{\rho_{j}}^{\nu)}\right) .
\end{aligned}
$$


An intriguing property of this vertex is that, should we set the energy on one of the external $z^{\mu}$ lines to zero, the resulting expression can also be obtained as a derivative of its lower-point cousin with respect to the impact factor:

$$
V_{\rho_{1} \ldots \rho_{n+1}}^{\mathrm{WL}, \mu \nu}\left(k ; \omega_{1}, \ldots, \omega_{n}, 0\right)=\frac{\partial}{\partial b^{\rho_{n+1}}} V_{\rho_{1} \ldots \rho_{n}}^{\mathrm{WL}, \mu \nu}\left(k ; \omega_{1}, \ldots, \omega_{n}\right) .
$$

This will be important when we return to the eikonal phase in section 7 .

\section{Radiation}

Having set up the worldline Feynman rules we begin with our first application: calculating the radiation far away from a source. For simplicity, let us first consider a single black hole. We calculate $k^{2}\left\langle h^{\mu \nu}(k)\right\rangle_{\mathrm{WQFT}}$ for $k^{2}=0$, where the expectation value of an operator in the WQFT was defined in eq. (3.30). This requires us to draw diagrams with a single outgoing graviton line, which is equivalent to solving Einstein's equation for $h_{\mu \nu}(x)$. For a single black hole

$$
-i k^{2}\left\langle h^{\mu \nu}(k)\right\rangle_{\mathrm{WQFT}}=-i \frac{m}{2 m_{\mathrm{Pl}}} e^{i k \cdot b} \delta(k \cdot v) v^{\mu} v^{\nu},
$$

which is simply the tadpole in eq. (4.12). ${ }^{15}$ In other words, $h_{\mu \nu}$ is directly sourced by the stress-energy tensor.

Like in section 3.1, we compare this with the three-point interaction vertex between two complex scalars $\varphi$ and a graviton $h_{\mu \nu}$ :

$$
\begin{aligned}
p \longrightarrow \overrightarrow{\xi_{\boldsymbol{y}}} p^{\prime} & =-i \kappa\left[p^{(\mu} p^{\nu)}-\frac{\eta^{\mu \nu}}{2}\left(p \cdot p^{\prime}-m^{2}\right)\right] \\
k & =-i \kappa\left[\hat{p}^{\mu} \hat{p}^{\nu}+\frac{1}{4}\left(\eta^{\mu \nu} k^{2}-k^{\mu} k^{\nu}\right)\right] .
\end{aligned}
$$

In the second equality we have inserted $p=\hat{p}+\frac{k}{2}, p^{\prime}=\hat{p}-\frac{k}{2}$, and using the mass-shell conditions $p^{2}=p^{\prime 2}=m^{2}$ we find that $\hat{p}^{2}=m^{2}-\frac{k^{2}}{4}$ and $\hat{p} \cdot k=0$. In the classical limit we write $k^{\mu}=\hbar \bar{k}^{\mu}$ and send $\hbar \rightarrow 0$, so we can discard the $k$-dependent terms. Finally inserting $\hat{p}^{\mu}=m v^{\mu},{ }^{16}$ we see that

$$
k^{2}\left\langle h^{\mu \nu}(k)\right\rangle_{\mathrm{WQFT}}=\frac{i}{2 m} e^{i k \cdot b} \delta(k \cdot v) \lim _{\hbar \rightarrow 0} \mathcal{M}_{\mathrm{GR}}^{\mu \nu}(p, k) .
$$

The graviton from a single black hole is therefore identified with the three-point amplitude (with the polarization tensor $\epsilon_{\mu \nu}$ stripped away).

This identity follows naturally from our discussion in section 2 and 3 . The exponential and $\delta$-function factors come from the central relationship in eq. (3.29); the factor of $\frac{1}{2 m}$

\footnotetext{
${ }^{15}$ Contracting with $P_{\mu \nu ; \rho \sigma}$ on an outgoing on-shell graviton line is unnecessary due to the tracelessness of the polarization tensor.

${ }^{16}$ Strictly speaking, we should write $\hat{p}^{\mu}=\hat{m} \hat{v}^{\mu}$ to represent the average momentum. However, at this low $\mathrm{PM}$ order the difference is inconsequential.
} 
from replacing $\sigma=\frac{\tau}{2 m}$. The interpretation of a single black hole radiating a graviton as a massive three-point amplitude has been widely studied elsewhere, including for higher spins $[92,93]$. The non-spinning black hole is associated with the Schwarzschild solution; a spinning black hole with the Kerr solution [125]. The corresponding double copies are closely related to the so-called Kerr-Schild double copy [126-128].

\subsection{Two-body radiation $(2 \mathrm{PM})$}

Let us now examine the radiation emitted from the inelastic scattering of a pair of nonspinning black holes at leading order (2PM). To begin with consider the five-point scattering amplitude:

$$
\begin{aligned}
& \epsilon_{\mu \nu} \mathcal{M}_{\mathrm{GR}}^{\mu \nu}\left(p_{i}, p_{i}^{\prime}, k\right)=p_{p_{2}}^{p_{1}}
\end{aligned}
$$

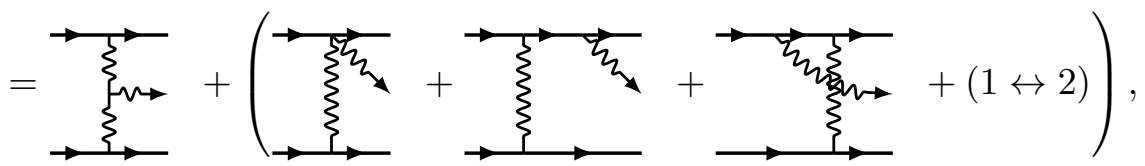

where $\epsilon_{\mu \nu}$ is the polarization of the emitted graviton with momentum $k$, and $p_{i}^{\prime}=p_{i}-q_{i}$. The on-shell conditions $\left(\hat{p}_{i} \pm \frac{q_{i}}{2}\right)^{2}=m_{i}^{2}$ imply $\hat{p}_{i} \cdot q_{i}=0$; momentum conservation gives $k=q_{1}+q_{2}$. Inserting the established relation (3.29) between an $n$-graviton form factor and a WQFT correlator into a generic $\phi_{1} \phi_{2} \rightarrow \phi_{1} \phi_{2}(+h)$ scattering amplitude à la eq. (2.19) yields a direct link to the WQFT:

$$
k^{2}\left\langle h^{\mu \nu}(k)\right\rangle_{\mathrm{WQFT}}=\frac{i}{4 m_{1} m_{2}} \int_{q_{1}, q_{2}} \hat{\mu}_{1,2}(k) \lim _{\hbar \rightarrow 0} \mathcal{M}_{\mathrm{GR}}^{\mu \nu}\left(p_{i}, p_{i}^{\prime}, k\right) .
$$

We have introduced the integral measure emerging from eq. (3.29):

$$
\hat{\mu}_{1,2}(k)=e^{i\left(q_{1} \cdot \hat{b}_{1}+q_{2} \cdot \hat{b}_{2}\right)} \delta\left(q_{1} \cdot \hat{v}_{1}\right) \delta\left(q_{2} \cdot \hat{v}_{2}\right) \delta\left(k-q_{1}-q_{2}\right) .
$$

This connection was already established in refs. [84, 129]; here we show how individual diagrams may be identified between the two methods. There are three diagrams in the WQFT, all with a single outgoing graviton line.

The first diagram contributing to $k^{2}\left\langle h^{\mu \nu}(k)\right\rangle_{\mathrm{WQFT}}$ is

$$
q_{1} \downarrow \sum_{q_{2}} \uparrow \sum_{i n \rightarrow, \nu}^{k}=-\frac{m_{1} m_{2}}{8 m_{\mathrm{Pl}}^{3}} \int_{q_{1}, q_{2}} \hat{\mu}_{1,2}(k) V_{3}^{(\mu \nu)(\rho \sigma)(\lambda \tau)} \frac{P_{\rho \sigma ; \alpha \beta}}{q_{1}^{2}} \frac{P_{\lambda \tau ; \gamma \delta}}{q_{2}^{2}} \hat{v}_{1}^{\alpha} \hat{v}_{1}^{\beta} \hat{v}_{2}^{\gamma} \hat{v}_{2}^{\delta},
$$

where $(i / 2) m_{\mathrm{Pl}}^{-1} V_{3}^{(\mu \nu)(\rho \sigma)(\lambda \tau)}\left(k,-q_{1},-q_{2}\right)$ is the three-graviton vertex. The delta functions in the measure $\hat{\mu}_{1,2}(k)$ are picked up from the vertices; we integrate over the intermediate 
momenta $q_{i}$. The counterpart to this diagram in $\mathcal{M}_{\mathrm{GR}}^{\mu \nu}$ is the first diagram in eq. (5.4), so we simply re-interpret the worldlines as scalars. Showing that the two expressions match in the $\hbar \rightarrow 0$ limit is trivial: the graviton propagators and three-graviton vertex are the same in either case, and we have already shown in eq. (5.2) that when $\hbar \rightarrow 0$ the scalarscalar-graviton vertex maps onto the stress-energy tensor.

A more interesting comparison is with this diagram:

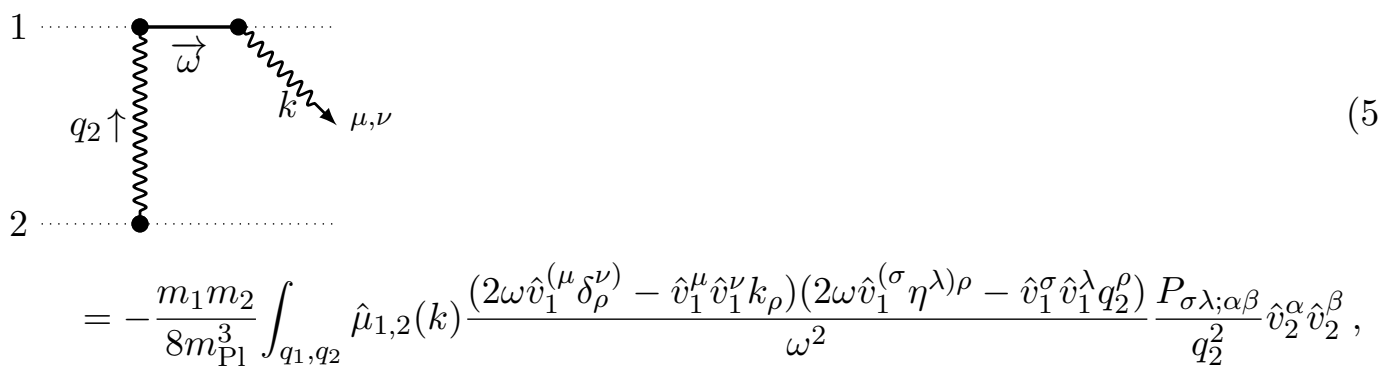

where $\omega=\hat{v}_{1} \cdot k=\hat{v}_{1} \cdot q_{2}$ from the $\delta$-function constraints. We have have massaged the integral measure into $\hat{\mu}_{1,2}(k)$ by performing the trivial $\omega$ integration:

$$
\int_{\omega, q_{2}} e^{i\left(\hat{b}_{1} \cdot\left(k-q_{2}\right)+\hat{b}_{2} \cdot q_{2}\right)} \delta\left(\hat{v}_{2} \cdot q_{2}\right) \delta\left(\omega-\hat{v}_{1} \cdot q_{2}\right) \delta\left(\omega-\hat{v}_{1} \cdot k\right)=\int_{q_{1}, q_{2}} \hat{\mu}_{1,2}(k) .
$$

This expression arises from the classical limit of three diagrams in $\mathcal{M}_{\mathrm{GR}}^{\mu \nu}$, again drawn in eq. (5.4). We can intuitively see where the $1 / \omega^{2}$ factor comes from by studying the classical limit of the scalar propagators:

$$
\begin{aligned}
& \frac{i}{\left(\hat{p}_{1}+\frac{q_{1}}{2}+q_{2}\right)^{2}-m_{1}^{2}}=\frac{i}{2 \hat{p}_{1} \cdot q_{2}+q_{2} \cdot k}=\frac{i}{2 \hat{p}_{1} \cdot q_{2}}\left(1-\frac{q_{2} \cdot k}{2 \hat{p}_{1} \cdot q_{2}}+\cdots\right), \\
& \frac{i}{\left(\hat{p}_{1}-\frac{q_{1}}{2}-q_{2}\right)^{2}-m_{1}^{2}}=\frac{-i}{2 \hat{p}_{1} \cdot q_{2}-q_{2} \cdot k}=\frac{-i}{2 \hat{p}_{1} \cdot q_{2}}\left(1+\frac{q_{2} \cdot k}{2 \hat{p}_{1} \cdot q_{2}}+\cdots\right),
\end{aligned}
$$

having used $\left(\hat{p}_{1} \pm \frac{q_{1}}{2}\right)^{2}=m_{1}^{2}$. The leading terms cancel when added; the sub-leading terms give rise to the desired $1 / \omega^{2}$ propagator.

The third diagram is related to the previous one by symmetry. Adding up the contributions and dropping unnecessary terms we get $^{17}$

$$
\begin{aligned}
k^{2}\left\langle h^{\mu \nu}(k)\right\rangle_{\mathrm{WQFT}}= & \frac{m_{1} m_{2}}{4 m_{\mathrm{Pl}}^{3}} \int_{q_{1}, q_{2}} \hat{\mu}_{1,2}(k) \\
& \times\left[\frac{\hat{P}_{12}^{(\mu}\left(\hat{P}_{12}^{\nu)}+\hat{\gamma} \hat{Q}_{12}^{\nu)}\right)}{q_{1}^{2} q_{2}^{2}}+\frac{2 \hat{\gamma}^{2}-1}{8}\left(\frac{\hat{Q}_{12}^{\mu} \hat{Q}_{12}^{\nu}}{q_{1}^{2} q_{2}^{2}}-\frac{\hat{P}_{12}^{\mu} \hat{P}_{12}^{\nu}}{\left(k \cdot \hat{v}_{1}\right)^{2}\left(k \cdot \hat{v}_{2}\right)^{2}}\right)\right],
\end{aligned}
$$

where $\hat{\gamma}=\hat{v}_{1} \cdot \hat{v}_{2}$ and we have recycled some notation from ref. [84]:

$$
\begin{aligned}
& \hat{P}_{12}^{\mu}=k \cdot \hat{v}_{1} \hat{v}_{2}^{\mu}-k \cdot \hat{v}_{2} \hat{v}_{1}^{\mu}, \\
& \hat{Q}_{12}^{\mu}=\left(q_{1}-q_{2}\right)^{\mu}-\frac{q_{1}^{2}}{k \cdot \hat{v}_{1}} \hat{v}_{1}^{\mu}+\frac{q_{2}^{2}}{k \cdot \hat{v}_{2}} \hat{v}_{2}^{\mu} .
\end{aligned}
$$

These vectors satisfy $\hat{P}_{12} \cdot k=\hat{Q}_{12} \cdot k=0$, which makes gauge invariance manifest.

\footnotetext{
${ }^{17}$ In the full metric $g_{\mu \nu}=\eta_{\mu \nu}+m_{\mathrm{Pl}}^{-1} h_{\mu \nu}$ the radiation occurs at $\mathcal{O}\left(m_{\mathrm{Pl}}^{-4}\right)=\mathcal{O}\left(G^{2}\right)$, i.e.2PM.
} 


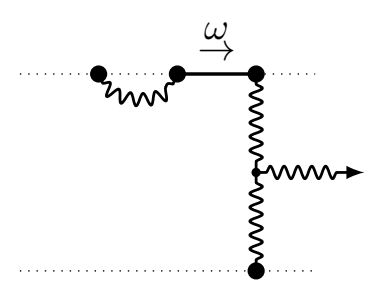

Figure 2. An example of a self-energy diagram, which does not appear in a three-body calculation at the same PM order. On support of the $\delta$-function constraints we have $\omega=0$, which gives rise to a singularity in the $1 / \omega^{2}$ propagator.

As $\mathcal{M}_{\mathrm{GR}}^{\mu \nu}$ consists of Feynman propagators (both for internal gravitons and scalars) using the established link to compute $k^{2}\left\langle h^{\mu \nu}(k)\right\rangle_{\text {WQFT }}$ gives rise to Feynman-type graviton propagators and time-symmetric $1 / \omega^{2}$ worldline propagators in the classical $\hbar \rightarrow 0$ limit. In the latter case, one can re-examine eq. (5.10) and carefully track the $i \epsilon$ 's through the calculation to show that the result is an average of the advanced/retarded propagators given in eq. (4.7). This is consistent with our use of $\hat{b}_{i}^{\mu}$ and $\hat{v}_{i}^{\mu}$ - as we discussed at the end of section 3.4, the choice of propagators corresponds to picking a specific inhomogeneous solution to the equations of motion.

In a genuine physical setting one might also wish to describe the radiation in terms of $b_{i}^{\mu}$ and $v_{i}^{\mu}$, corresponding to the initial trajectories of the black holes. This would require the use of retarded propagators for the worldline fluctuations in the above calculation, which should always point towards the outgoing graviton and thus provide a clear flow of causality. In the WQFT one could simply adopt these propagators from the start; if using an amplitude and taking the classical limit one should take care and change the $i \epsilon$ prescription before integration.

While for the worldlines the integration constants $b_{i}^{\mu}, v_{i}^{\mu}$ mediate between different propagator choices, this possibility is not available for the gravitons: a retarded propagator is demanded by the physical setup. Strictly speaking, the expectation value $k^{2}\left\langle h^{\mu \nu}(k)\right\rangle_{\mathrm{WQFT}}$ as defined above in terms of a path integral (3.30) leads to Feynman graviton propagators ${ }^{18}$ — but of course the $i \epsilon$ prescription can also be adapted for the gravitons before integration by identifying the flow of causality.

\subsection{Three-body radiation $(3 \mathrm{PM})$}

At 3PM we find it convenient to first study the three-body problem, and the specialization to the two-body problem should just be a special case. ${ }^{19} \mathrm{~A}$ three-body starting point was also used in ref. [135] to study two-body radiation in dilaton gravity, and proved helpful when considering the double copy. It allows us to identify additional symmetries of the

\footnotetext{
${ }^{18}$ This is rooted in the fact that this expectation value uses a state fixed by boundary conditions ("inout") instead of initial conditions ("in-in") — see refs. [130, 131] for a discussion in the worldline EFT context. See also ref. [86] for a derivation of radiation reaction effects from amplitudes, which involves terms quadratic in the Feynman-propagator based amplitudes.

${ }^{19}$ The two-body waveform at this order was previously discussed in the context of soft theorems from an amplitudes perspective [132, 133] (see also ref. [134]).
} 


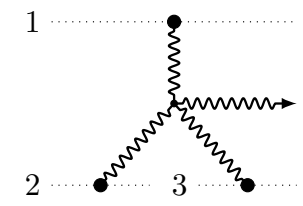

(a) $S_{a}=6$

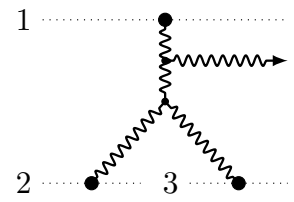

(b) $S_{b}=2$

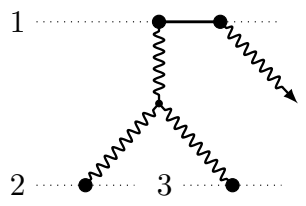

(c) $S_{c}=2$

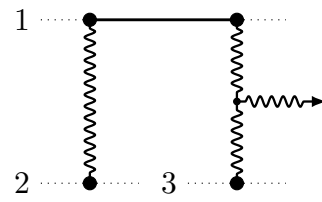

(d) $S_{d}=1$

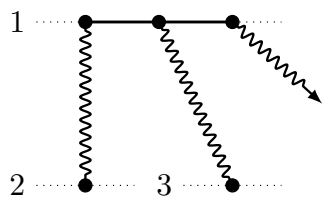

(e) $S_{e}=1$

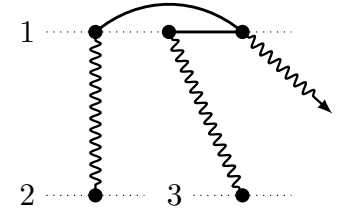

(f) $S_{f}=2$

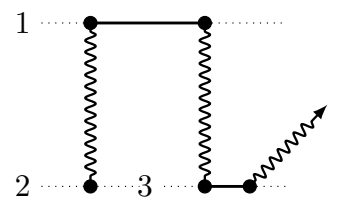

(g) $S_{g}=1$

Figure 3. The seven diagrams contributing to the radiation $k^{2}\left\langle h^{\mu \nu}(k)\right\rangle_{\mathrm{WQFT}}$ at order 3PM, and their respective symmetry factors. All seven graphs represent tree-level contributions, the worldlines being drawn only as a visual aid. The outgoing momentum from each worldline is $q_{i}^{\mu}$.

diagrams, and avoid self-energy graphs which would otherwise give singularities in the two-body problem. An example of such an unwanted graph is given in figure 2 .

The radiation is fully described by the seven diagrams in figure 3 :

$$
k^{2}\left\langle h^{\mu \nu}(k)\right\rangle_{\mathrm{WQFT}}=\frac{m_{1} m_{2} m_{3}}{m_{\mathrm{Pl}}^{5}} \sum_{S_{3}} \sum_{i \in\{a, \ldots, g\}} \int_{q_{1}, q_{2}, q_{3}} \mu_{1,2,3}(k) \frac{1}{S_{i}} \frac{N_{i}^{\mu \nu}}{D_{i}},
$$

where we now use retarded propagators both for the gravitons and worldline (but omit the propagator on the outgoing line with momentum $k^{\mu}$ ). As the diagrams must connect with all three worldlines, self-energy diagrams of the kind in figure 2 are avoided. We sum over 3 ! permutations of the worldlines, swapping $q_{i}^{\mu}, b_{i}^{\mu}$ and $v_{i}^{\mu}$ in each case. By design these permutations preserve the integral measure emerging from eq. (3.29):

$$
\mu_{1,2,3}(k)=e^{i\left(q_{1} \cdot b_{1}+q_{2} \cdot b_{2}+q_{3} \cdot b_{3}\right)} \delta\left(v_{1} \cdot q_{1}\right) \delta\left(v_{2} \cdot q_{2}\right) \delta\left(v_{3} \cdot q_{3}\right) \delta\left(k-q_{1}-q_{2}-q_{3}\right),
$$

which (after an appropriate rearrangement) is the same for all seven diagrams. Each symmetry factor $S_{i}$ corrects for an "overcount" in the sum - for example, diagram (a) is invariant under all 6 permutations, so dividing by $S_{a}=6$ accounts for this. The propagator factors are

$$
\begin{array}{ll}
D_{a}=q_{1}^{2} q_{2}^{2} q_{3}^{2}, & D_{b}=q_{1}^{2} q_{2}^{2} q_{3}^{2} q_{23}^{2}, \\
D_{c}=q_{2}^{2} q_{3}^{2} q_{23}^{2}\left(\omega_{1}+i \epsilon\right)^{2}, & D_{d}=q_{2}^{2} q_{3}^{2} q_{12}^{2}\left(\omega_{12}+i \epsilon\right)^{2}, \\
D_{e}=q_{2}^{2} q_{3}^{2}\left(\omega_{1}+i \epsilon\right)^{2}\left(\omega_{12}+i \epsilon\right)^{2}, & D_{f}=q_{2}^{2} q_{3}^{2}\left(\omega_{12}+i \epsilon\right)^{2}\left(\omega_{13}+i \epsilon\right)^{2}, \\
D_{g}=q_{2}^{2} q_{12}^{2}\left(\omega_{12}+i \epsilon\right)^{2}\left(\omega_{3}+i \epsilon\right)^{2}, &
\end{array}
$$

where $q_{i j}=q_{i}+q_{j}$ and we have introduced new variables:

$$
\omega_{i}=v_{i} \cdot k
$$$$
\omega_{i j}=v_{i} \cdot q_{j}
$$ 
On support of $\mu_{1,2,3}(k), \omega_{i}=\sum_{j=1}^{3} \omega_{i j}$ and $\omega_{i i}=0$ (no sum on $i$ ). To confirm this result we have checked the off-shell $\left(k^{2} \neq 0\right)$ Ward identity

$$
k_{\mu}\left\langle h^{\mu \nu}(k)\right\rangle_{\mathrm{WQFT}}=0,
$$

which holds already at the integrand level.

Our final result for $k^{2}\left\langle h^{\mu \nu}(k)\right\rangle_{\mathrm{WQFT}}$ is presented in the supplementary material of this paper, with expressions given for each of the seven numerators $N_{i}^{\mu \nu}$ in figure 3. In a separate Mathematica file we also explicitly demonstrate the off-shell Ward identity; ${ }^{20}$ the on-shell graviton can easily be obtained by setting $k^{2}=0$. We claim that the same integrand (with $b_{i}^{\mu} \rightarrow \hat{b}_{i}^{\mu}, v_{i}^{\mu} \rightarrow \hat{v}_{i}^{\mu}$ ) can also be obtained from a seven-point scalar-graviton amplitude with three pairs of distinctly flavored scalars:

$$
k^{2}\left\langle h^{\mu \nu}(k)\right\rangle_{\mathrm{WQFT}}=\frac{i}{8 m_{1} m_{2} m_{3}} \int_{q_{1}, q_{2}, q_{3}} \hat{\mu}_{1,2,3}(k) \lim _{\hbar \rightarrow 0} \mathcal{M}_{\mathrm{GR}}^{\mu \nu}\left(p_{i}, p_{i}^{\prime}, k\right),
$$

but checking this explicitly we save for future work.

\section{Deflections}

Let us now switch to a purely conservative setting. We compute the impulse on a single black hole in a binary scattering, which classically can be expressed as

$$
\Delta p_{i}^{\mu}=m_{i} \Delta \dot{x}_{i}^{\mu}=m_{i} \int \mathrm{d} \dot{x}_{i}^{\mu}\left(\tau_{i}\right)=m_{i} \int_{-\infty}^{\infty} \mathrm{d} \tau_{i} \frac{\mathrm{d}^{2} z_{i}^{\mu}\left(\tau_{i}\right)}{\mathrm{d} \tau_{i}^{2}} .
$$

Even though this is a total derivative, in the present context it does not integrate to zero. In the WQFT (where $z_{i}^{\mu}\left(\tau_{i}\right)$ is promoted to an operator) our task is therefore to calculate the expectation value

$$
\Delta p_{i}^{\mu}=m_{i} \int_{-\infty}^{\infty} \mathrm{d} \tau_{i}\left\langle\frac{\mathrm{d}^{2} z_{i}^{\mu}\left(\tau_{i}\right)}{\mathrm{d} \tau_{i}^{2}}\right\rangle_{\mathrm{WQFT}} .
$$

Inserting the Fourier space definition of $z^{\mu}(4.1)$ the impulse becomes

$$
\Delta p_{i}^{\mu}=m_{i} \int_{\omega}(-i \omega)^{2}\left\langle z_{i}^{\mu}(\omega) \delta(\omega)\right\rangle_{\mathrm{WQFT}}=-\left.m_{i} \omega^{2}\left\langle z_{i}^{\mu}(\omega)\right\rangle_{\mathrm{WQFT}}\right|_{\omega=0} .
$$

Hence the impulse follows from drawing tree-level graphs with a cut external $z_{i}^{\mu}$ line, multiplied by a factor of $i$ for the correct normalization. This is analogous to how we computed $k^{2}\left\langle h^{\mu \nu}(k)\right\rangle_{\mathrm{WQFT}}$ with $k^{2}=0$ in section 5 . By using retarded worldline propagators we ensure a flow of causality towards the outgoing line; time-symmetric Feynman propagators for the gravitons imply a purely elastic scattering of the black holes. To include radiative effects one could instead use retarded graviton propagators, but in the integrals that follow we shall assume the former.

As a demonstration, we shall now compute the conservative deflection $\Delta p_{1}^{\mu}$ up to order 2PM, specifically reproducing the integrands by Kälin and Porto [65] whose integrated result matches earlier work by e.g. Westpfahl [12]. Our method does not require the determination of an effective action for the black holes.

\footnotetext{
${ }^{20}$ Our demonstration file relies on the tensor computer algebra package xAct [136].
} 


\subsection{Leading order (1PM)}

At leading order $\Delta p_{1}^{\mu}$ is described by a single diagram:

$$
2 \sum_{q \uparrow}^{1 \cdots=0}=i \frac{m_{1} m_{2}}{4 m_{\mathrm{Pl}}^{2}} \int_{q} e^{i q \cdot b} \delta\left(q \cdot v_{1}\right) \delta\left(q \cdot v_{2}\right)\left(-v_{1}^{\nu} v_{1}^{\rho} q^{\mu}\right) \frac{P_{\nu \rho ; \sigma \lambda}}{q^{2}} v_{2}^{\sigma} v_{2}^{\lambda},
$$

where $b^{\mu}=b_{2}^{\mu}-b_{1}^{\mu}\left(b^{2}<0\right)$. Cleaning this up we deduce that

$$
\Delta p_{1}^{\mu}=\frac{m_{1} m_{2}}{8 m_{\mathrm{Pl}}^{2}}\left(2 \gamma^{2}-1\right) \frac{\partial}{\partial b_{1, \mu}} \int_{q} \frac{\delta\left(q \cdot v_{1}\right) \delta\left(q \cdot v_{2}\right)}{q^{2}} e^{i q \cdot b},
$$

where $\gamma=v_{1} \cdot v_{2}>1$. This matches eq. (4.9) in ref. [65].

The integral above can be performed in a variety of ways (see also e.g.ref. [137]); to maintain covariance we find it convenient to decompose $q=q_{\|}+q_{\perp}$, where $q_{\|}=P_{\|} q$ is parallel and $q_{\perp}=P_{\perp} q$ is perpendicular to the plane described by the two-form $P=v_{1} \wedge v_{2} \cdot{ }^{21}$ The projectors $P_{\|}$and $P_{\perp}$ are

$$
\begin{aligned}
& P_{\|}^{\mu \nu}=\frac{P^{\mu \rho} P_{\rho}^{\nu}}{|P|^{2}}=-\frac{1}{\gamma^{2}-1}\left[v_{1}^{\mu} v_{1}^{\nu}-2 \gamma v_{1}^{(\mu} v_{2}^{\nu)}+v_{2}^{\mu} v_{2}^{\nu}\right], \\
& P_{\perp}^{\mu \nu}=\eta^{\mu \nu}-P_{\|}^{\mu \nu},
\end{aligned}
$$

where $|P|^{2} \equiv-\frac{1}{2} P_{\mu \nu} P^{\mu \nu}=\gamma^{2}-1 . \quad P_{\| \mu \nu}$ is the induced metric of the parallel plane, so we adopt the notation $\eta_{\|}^{\mu \nu} \equiv P_{\|}^{\mu \nu}$; the corresponding volume form is $\epsilon_{\| \mu \nu}=-\epsilon_{\| \nu \mu}$. It holds that $\operatorname{det}_{\|} \eta_{\|}=-1$ and $\epsilon_{\|}^{\mu \nu} \epsilon_{\|}^{\alpha \beta}=-\eta_{\|}^{\mu \alpha} \eta_{\|}^{\nu \beta}+\eta_{\|}^{\mu \beta} \eta_{\|}^{\nu \alpha}$. The Dirac deltas impose $q_{\|} \cdot v_{1}=q_{\|} \cdot v_{2}=0$, and upon eliminating them we get an additional factor from the Jacobian determinant:

$$
\delta(\underbrace{v_{1} \cdot q_{\|}}_{x}) \delta(\underbrace{v_{2} \cdot q_{\|}}_{y})=\underbrace{\left|\frac{\partial(x, y)}{\partial q_{\|}}\right|^{-1}}_{\operatorname{det}_{\|}^{-1}\left(v_{1}, v_{2}\right)=|P|^{-1}} \delta\left(q_{\|}^{\mu}\right),
$$

where $\operatorname{det}_{\|}\left(v_{1}, v_{2}\right)=\frac{1}{2} \epsilon_{\|}^{\mu \nu} P_{\mu \nu}$ and $\left(\epsilon_{\|}^{\mu \nu} P_{\mu \nu}\right)^{2}=-2 P_{\mu \nu} P^{\mu \nu}=4|P|^{2}$. Therefore

$$
\int_{q} \frac{\delta\left(q \cdot v_{1}\right) \delta\left(q \cdot v_{2}\right)}{q^{2}} e^{i q \cdot b}=\frac{1}{|P|} \int_{q_{\perp}} \frac{e^{i q_{\perp} \cdot b}}{q_{\perp}^{2}}=\frac{\log |b|}{2 \pi|P|}+\text { const }
$$

and when plugged into eq. (6.5) this yields the final result: ${ }^{22}$

$$
\Delta p_{1}^{\mu}=2 G m_{1} m_{2} \frac{\left(2 \gamma^{2}-1\right)}{\sqrt{\gamma^{2}-1}} \frac{b^{\mu}}{\left|b^{2}\right|},
$$

where $m_{\mathrm{Pl}}^{-1}=\sqrt{32 \pi G}$. This result can also be found in (for example) refs. [78, 93].

\footnotetext{
${ }^{21}$ We thank Gregor Kälin and Rafael Porto for sharing details of this method with us.

${ }^{22}$ The overall sign is consistent with an attractive gravitational force: $\Delta p_{1}^{\mu}$ aligns with $b^{\mu}=b_{2}^{\mu}-b_{1}^{\mu}$, which points from the first to the second black hole.
} 


\subsection{Sub-leading order $(2 \mathrm{PM})$}

At $\mathcal{O}\left(G^{2}\right)$ there are four contributing diagrams, with two each proportional to $m_{1} m_{2}^{2}$ and $m_{1}^{2} m_{2}$. As they carry different integral measures we treat them separately. In the first category:

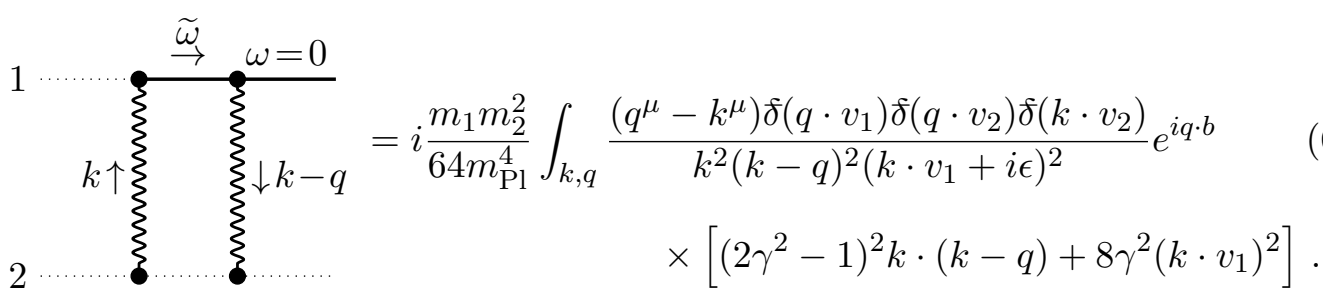

The integral measure is $\int_{k, q, \tilde{\omega}} \delta\left(k \cdot v_{2}\right) \delta\left(\tilde{\omega}-k \cdot v_{1}\right) \delta\left(\tilde{\omega}-(k-q) \cdot v_{1}\right) \delta\left((q-k) \cdot v_{2}\right)$ in its initial form; $\tilde{\omega}$ integration yields $\widetilde{\omega}=k \cdot v_{1}$ and leaves the three remaining $\delta$-functions in eq. (6.10). This diagram matches eq. (4.14) of ref. [65], up to terms that vanish upon integration (those that do not contribute to long-range interactions). The other diagram with the same integral measure is

$$
\begin{gathered}
1 \\
2 \cdots
\end{gathered}
$$

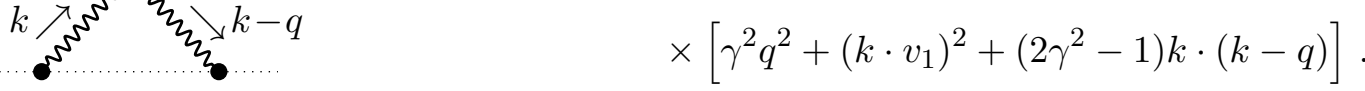

This agrees with eq. (4.15) of ref. [65] (up to a symmetry factor of $1 / 2$ ). In the second category we have

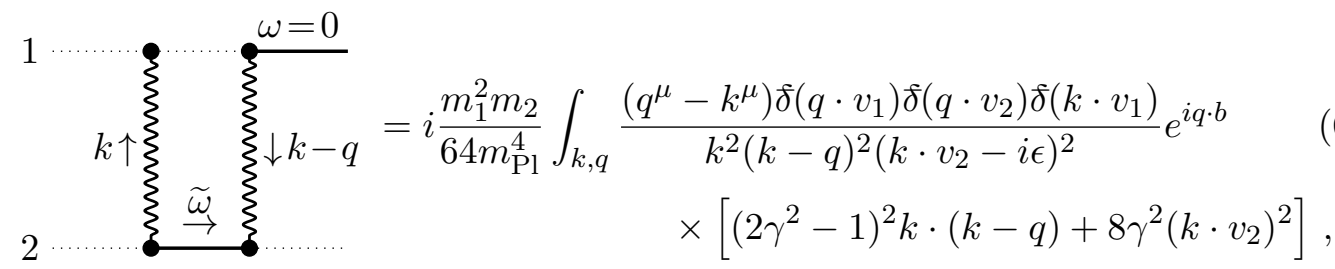

which (except for the outgoing $\omega=0$ line) is related to (6.10) by symmetry; the $\delta$-function constraint yields $\tilde{\omega}=-k \cdot v_{2}$. Finally,

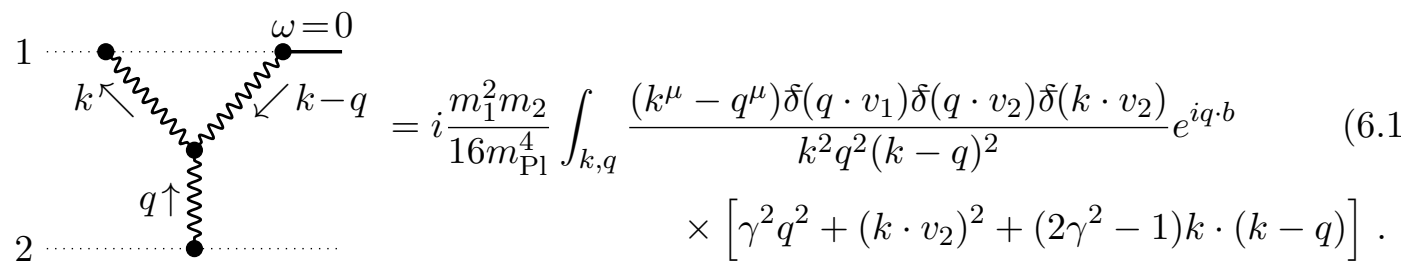

Not included are diagrams involving self-interactions of gravitons on a single worldline, which also do not contribute to the final integrated result.

Taken together, these four diagrams make up the 2PM deflection. As the integration was already discussed at length in ref. [65] we will not reiterate the details; instead we 
simply present the final result for the conservative impulse at this order:

$$
\begin{aligned}
\Delta p_{1}^{\mu}= & \frac{G m_{1} m_{2} b^{\mu}}{\left|b^{2}\right|}\left(\frac{2\left(2 \gamma^{2}-1\right)}{\sqrt{\gamma^{2}-1}}+\frac{3 \pi}{4} \frac{\left(5 \gamma^{2}-1\right)}{\sqrt{\gamma^{2}-1}} \frac{G\left(m_{1}+m_{2}\right)}{|b|}\right) \\
& -2 G^{2} m_{1} m_{2} \frac{\left(2 \gamma^{2}-1\right)^{2}}{\left(\gamma^{2}-1\right)^{2}\left|b^{2}\right|}\left(\left(\gamma m_{1}+m_{2}\right) v_{1}^{\mu}-\left(\gamma m_{2}+m_{1}\right) v_{2}^{\mu}\right) .
\end{aligned}
$$

This includes the 1PM result already given in eq. (6.9), and agrees with Westpfahl's result [12]. It also satisfies

$$
p_{1}^{2}=\left(p_{1}+\Delta p_{1}\right)^{2}
$$

up to terms $\mathcal{O}\left(G^{3}\right)$, using $b \cdot v_{i}=0$. This is consistent with our use of retarded worldline propagators: $p_{i}^{\mu}=m_{i} v_{i}^{\mu}$ are the incoming momenta.

Should we flip the sign on $i \epsilon$ throughout our calculation above, i.e.use advanced instead of retarded worldline propagators, then the result (6.14) in terms of $b_{i}^{\prime \mu}$ and $v_{i}^{\prime \mu}$ is identical except with the signs on $v_{i}^{\prime \mu}$ reversed. This is consistent with the impulse instead obeying $p_{1}^{\prime 2}=\left(p_{1}^{\prime}-\Delta p_{1}\right)^{2}$, where $p_{i}^{\prime \mu}=m_{i} v_{i}^{\prime \mu}$ are the outgoing momenta. Similarly, if we use time-symmetric worldline propagators (an average of advanced/retarded) then the terms proportional to $\hat{v}_{i}^{\mu}$ vanish altogether - more on this in the next section. The impulse obeys $\left(\hat{p}_{1}+\frac{\Delta p_{1}}{2}\right)^{2}=\left(\hat{p}_{1}-\frac{\Delta p_{1}}{2}\right)^{2}$, i.e. $\hat{p}_{1} \cdot \Delta p_{1}=0$.

\section{Eikonal phase}

Having now computed the deflection $\Delta p_{1}^{\mu}$ up to $2 \mathrm{PM}$ order, let us finally explain its connection to scattering amplitudes. Unlike with the emitted graviton $k^{2}\left\langle h^{\mu \nu}(k)\right\rangle$ computed in section 5 , it is not immediately obvious how to obtain $\Delta p_{i}^{\mu}$ from an amplitudes perspective. The reason is simple: unlike $h_{\mu \nu}(x)$, the worldline fluctuations $z_{i}^{\mu}(\tau)$ do not live in the amplitudes; instead we have the scalars $\phi_{i}(x)$. So the trick we used in eq. (5.5) to integrate out the scalars in a five-point amplitude, leaving behind the expectation $\left\langle h^{\mu \nu}(k)\right\rangle$, does not work. Instead we need to make use of the four-point scalar amplitude $\mathcal{M}_{\phi_{1} \phi_{2} \rightarrow \phi_{1} \phi_{2}}$.

From an amplitudes perspective the eikonal phase of eq. (3.32) is a very useful scalar quantity, as it captures the impulse and other classical observables. Writing the S-matrix in terms of a scattering amplitude eq. (3.32) gives rise to

$$
\begin{aligned}
e^{i \chi} & =1+\frac{i}{4 m_{1} m_{2}} \int_{q} e^{i q \cdot b} \delta\left(v_{1} \cdot q\right) \delta\left(v_{2} \cdot q\right) \lim _{\hbar \rightarrow 0} \mathcal{M}_{\phi_{1} \phi_{2} \rightarrow \phi_{1} \phi_{2}}(q) \\
& =1+i \int_{q_{\perp}} e^{i q_{\perp} \cdot b} \frac{\mathcal{M}_{\phi_{1} \phi_{2} \rightarrow \phi_{1} \phi_{2}}(q)}{4 m_{1} m_{2} \sqrt{\gamma^{2}-1}}
\end{aligned}
$$

where $\chi$ is the eikonal phase and $q$ is the transferred momentum. It was demonstrated in refs. [85, 95] (see also ref. [65]) that the 2PM deflection in the center-of-mass system is obtained via

$$
\Delta p_{\perp}=\frac{\partial \chi}{\partial b}
$$

where $p_{\perp}=P_{\perp} p_{2}=-P_{\perp} p_{1}$. A similar relation holds for the scattering angle [85] - see also eq. (4.5) in ref. [98]. It is suggestive that such a relation can be extended to higher 
orders and changes in spins [94, 95]. That is, the eikonal phase $\chi$ appears to be a generator for all observables of conservative classical scattering. It can also be related to bound orbits via analytic continuation [138], analogously to the investigation in refs. [100, 101].

From our WQFT perspective we have shown in section 3.4 that the classical part of the eikonal phase $\chi$ is given by the free energy of the WQFT at tree level (integrating out the $z^{\mu}$ and $h_{\mu \nu}$ fields). We use Feynman propagators for the gravitons (which also occur in the QFT S-matrix) and time-symmetric propagators for the worldlines. So $\hat{b}_{i}^{\mu}$ and $\hat{v}_{i}^{\mu}$ are identified with the average of the incoming/outgoing momenta $\hat{p}_{i}^{\mu}$. Recalling eqs (3.31)-(3.33), the eikonal phase is then

$$
\begin{aligned}
e^{i \chi\left(\hat{b}_{i}, \hat{v}_{i}\right)} & =Z_{\mathrm{WQFT}} \\
& =\text { const } \times \int \mathcal{D}\left[h_{\mu \nu}, z_{1}^{\mu}, z_{2}^{\mu}\right] \exp \left[i\left(S_{\mathrm{EH}}+S_{\mathrm{gf}}-\sum_{i=1}^{n} \int_{-\infty}^{\infty} \mathrm{d} \tau_{i} \frac{m_{i}}{2} g_{\mu \nu} \dot{x}_{i}^{\mu} \dot{x}_{i}^{\nu}\right)\right],
\end{aligned}
$$

where we have dropped the Lee-Yang ghost contributions which are irrelevant for the classical limit. Instead of eq. (7.2) we will demonstrate that

$$
\Delta p_{1, \mu}=i \frac{\partial}{\partial \hat{b}_{1}^{\mu}} \log Z_{\mathrm{WQFT}}=-\frac{\partial \chi}{\partial \hat{b}_{1}^{\mu}}
$$

holds in our formalism to all orders as a consequence of eq. (4.18). This should satisfy $\left(\hat{p}_{1}+\frac{\Delta p_{1}}{2}\right)^{2}=\left(\hat{p}_{1}-\frac{\Delta p_{1}}{2}\right)^{2}$, i.e. $\hat{p}_{1} \cdot \Delta p_{1}=0$. Note how connected amplitudes get mapped into WQFT diagrams in $\mathcal{Z}_{\mathrm{WQFT}}$ that are disconnected in general, and finally exponentiate into connected WQFT diagrams in $\chi$. This manifests the exponentiation of amplitudes in the classical limit.

Let us now prove eq. (7.4). On the one hand, from eq. (6.3) we have that

$$
\begin{aligned}
& \Delta p_{1}^{\sigma}=-\left.m_{1} \omega^{2}\left\langle z_{1}^{\mu}(\omega)\right\rangle_{\mathrm{WQFT}}\right|_{\omega=0} \\
& =-m_{1}\left\langle\omega^{2} z_{1}^{\sigma}(\omega) \exp [\overbrace{\int \sum_{n=0}^{\infty} \frac{1}{n !} V_{1, \rho_{1} \ldots \rho_{n}}^{\mathrm{WL}, \mu \nu}\left(k ; \omega_{1}, \ldots, \omega_{n}\right) h_{\mu \nu}(k) z_{1}^{\rho_{1}}\left(\omega_{1}\right) \ldots z_{1}^{\rho_{n}}}^{=:\left(\mathrm{WL}_{1}\right)}\right. \\
& \left.\left.+\left(\mathrm{WL}_{2}\right)+(\mathrm{GR})\right]\right\rangle\left._{\text {free WQFT }}\right|_{\omega=0},
\end{aligned}
$$

with $\langle\ldots\rangle_{\text {free WQFT }}$ denoting the vacuum expectation value of the non-interacting theory and (GR) the graviton vertices. This expression involves only connected diagrams, or Wick contractions. We perform all Wick contractions involving the external $z_{1}^{\sigma}(\omega)$, noting that contractions with an $n$-vertex on the worldline give an overall factor of $n$. Thereafter we set $\omega=0$, essentially canceling the external propagator:

$$
\begin{aligned}
\Delta p_{1}^{\sigma}=i\left\langle\left(\int \sum _ { n = 1 } ^ { \infty } \frac { 1 } { ( n - 1 ) ! } V _ { 1 , \rho _ { 1 } \ldots \rho _ { n } } ^ { \mathrm { WL } , \mu \nu } \left( k ; \omega_{1}, \ldots,\right.\right.\right. & \left.\left., \omega_{n-1}, \omega\right) h_{\mu \nu} z_{1}^{\rho_{1}} \ldots z_{1}^{\rho_{n-1}} \eta^{\sigma \rho_{n}}\right) \\
& \left.\times e^{\left(\mathrm{WL}_{1}\right)+\left(\mathrm{WL}_{2}\right)+(\mathrm{GR})}\right\rangle\left._{\text {free WQFT }}\right|_{\omega=0} .
\end{aligned}
$$


On the other hand, again considering connected contractions only (leaving the log implicit), it holds that

$$
\begin{aligned}
& \Delta p_{1}^{\sigma}=i \frac{\partial i \chi}{\partial b_{1, \sigma}} \\
& =i\left\langle\frac{\partial}{\partial b_{1, \sigma}} e^{\left(\mathrm{WL}_{1}\right)+\left(\mathrm{WL}_{2}\right)+(\mathrm{GR})}\right\rangle_{\text {free WQFT }} \\
& =i\left\langle(\int \sum_{n=0}^{\infty} \frac{1}{n !} \underbrace{\frac{\partial}{\partial b_{1, \sigma}} V_{1, \rho_{1} \ldots \rho_{n}}^{\mathrm{WL}, \mu \nu}\left(k ; \omega_{1}, \ldots, \omega_{n}\right)} h_{\mu \nu} z_{1}^{\rho_{1}} \ldots z_{1}^{\rho_{n}})\right.
\end{aligned}
$$

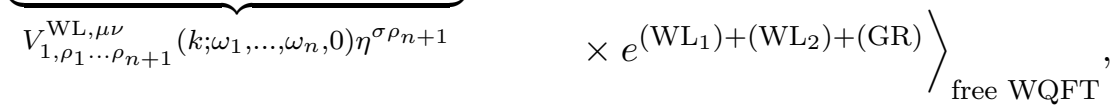

making crucial use of eq. (4.18). This is the same as the preceding equation when shifting $n$ and using $\omega=0$, thus showing eq. (7.4).

Let us now work out the eikonal to $2 \mathrm{PM}$ order. The corresponding diagrams in the eikonal phase are

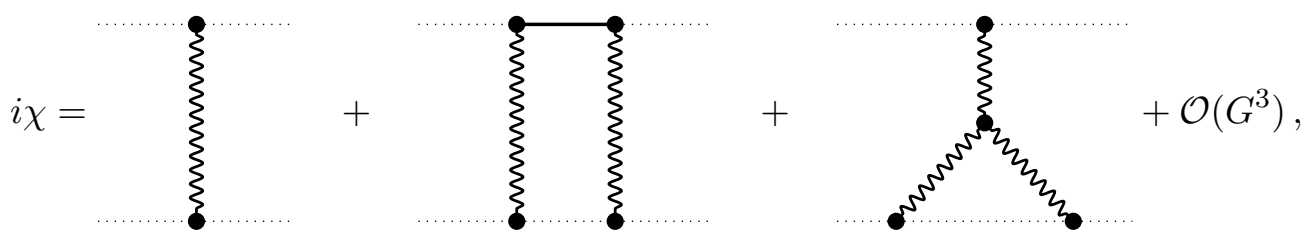

where mirror diagrams are left implicit. Assembling the contributions in (7.8) and performing the integrals one finds

$$
\chi=G m_{1} m_{2}\left(-\frac{2\left(2 \hat{\gamma}^{2}-1\right)}{\sqrt{\hat{\gamma}^{2}-1}} \log |\hat{b}|+\frac{3 \pi}{4} \frac{\left(5 \hat{\gamma}^{2}-1\right)}{\sqrt{\hat{\gamma}^{2}-1}} \frac{G\left(m_{1}+m_{2}\right)}{|\hat{b}|}\right)+\mathcal{O}\left(G^{3}\right),
$$

in agreement with ref. [85]. Taking the derivative with respect to $\hat{b}_{1}^{\mu}$ we obtain the impulse:

$$
\Delta p_{1}^{\mu}=\frac{G m_{1} m_{2} \hat{b}^{\mu}}{\left|\hat{b}^{2}\right|}\left(\frac{2\left(2 \hat{\gamma}^{2}-1\right)}{\sqrt{\hat{\gamma}^{2}-1}}+\frac{3 \pi}{4} \frac{\left(5 \hat{\gamma}^{2}-1\right)}{\sqrt{\hat{\gamma}^{2}-1}} \frac{G\left(m_{1}+m_{2}\right)}{|\hat{b}|}\right)+\mathcal{O}\left(G^{3}\right) .
$$

As it depends on $\hat{b}_{i}^{\mu}$ and $\hat{v}_{i}^{\mu}$, this expression is different from eq. (6.14) derived earlier (which depended on $b_{i}^{\mu}$ and $v_{i}^{\mu}$ ). It satisfies $\hat{p}_{1} \cdot \Delta p_{1}=0$ as expected.

One may naturally ask whether, having obtained the conservative deflection in eq. (7.10) in terms of $\hat{b}^{\mu}$ and $\hat{v}_{i}^{\mu}$, one could subsequently extract the (arguably more useful) result in eq. (6.14) involving involving $b^{\mu}$ and $v_{i}^{\mu}$. At this PM order we make certain simplifying assumptions: $\hat{v}_{i}^{\mu}=v_{i}^{\mu}+\mathcal{O}(G)$ implies $\hat{\gamma}=\gamma+\mathcal{O}\left(G^{2}\right)$. Also, $\hat{b}^{\mu}=b^{\mu}+x_{1} v_{1}^{\mu}+x_{2} v_{2}^{\mu}$ where $x_{i} \sim \mathcal{O}(G)$ and $b \cdot v_{i}=0$ implies $|\hat{b}|=|b|+\mathcal{O}\left(G^{2}\right)$. So there is no need to distinguish between different versions of $|b|$ and $\gamma$ at 2PM. To reproduce the result in eq. (6.14) we simply need to find the terms in the $v_{i}^{\mu}$ directions: it was demonstrated in ref. [70] that by demanding $p_{1}^{2}=\left(p_{1}+\Delta p_{1}\right)^{2}$ (to all orders in $G$ ) the missing terms are reproducible by iteration from lower orders. 
From the deflection $\Delta p_{1}^{\mu}$ we can also find the scattering angle $\theta$ (see e.g.refs. [85, 139, $140])$. In the center-of-mass $(\mathrm{COM})$ frame $p_{1}=\left(E_{1}, \mathbf{p}\right), p_{2}=\left(E_{2},-\mathbf{p}\right)$ and

$$
|\Delta \mathbf{p}|=2|\mathbf{p}| \sin \left(\frac{\theta}{2}\right)
$$

In the COM frame one can also deduce that

$$
|\mathbf{p}|=\frac{m_{1} m_{2} \sqrt{\gamma^{2}-1}}{\sqrt{m_{1}^{2}+m_{2}^{2}+2 m_{1} m_{2} \gamma}} .
$$

The total angular momentum is given by $J=|\mathbf{b} \times \mathbf{p}|=|\mathbf{b}||\mathbf{p}|$. Putting the pieces together, we find that

$$
\sin \left(\frac{\theta}{2}\right)=\frac{G m_{1} m_{2}}{J}\left(\frac{\left(2 \gamma^{2}-1\right)}{\sqrt{\gamma^{2}-1}}+\frac{3 \pi}{8} \frac{G m_{1} m_{2}\left(m_{1}+m_{2}\right)\left(5 \gamma^{2}-1\right)}{J \sqrt{m_{1}^{2}+m_{2}^{2}+2 m_{1} m_{2} \gamma}}\right)+\mathcal{O}\left(G^{3}\right)
$$

fully describes the scattering angle to order $2 \mathrm{PM}$.

\section{Discussion}

In this paper we have examined the link between scattering amplitudes and observables in a worldline quantum field theory (WQFT). The link is manifested by a worldline path integral representation of the graviton-dressed scalar propagator, which can be inserted into a formal definition of the S-matrix in terms of time-ordered correlators - formally integrating out the scalars on external lines. By taking the classical $\hbar \rightarrow 0$ limit we can interpret the results as expectation values of operators in the WQFT. Performing LSZ reduction on the time-ordered correlators, i.e.cutting the propagators on their external lines, corresponds with allowing the worldlines of each black hole to span an infinite proper time domain $\tau \in[-\infty, \infty]$. We also derived the crucial relationship in the classical $\hbar \rightarrow 0$ limit:

$$
\mathcal{Z}_{\mathrm{WQFT}}=e^{i \chi}
$$

i.e.the free energy of the WQFT corresponds precisely with the eikonal phase of a $2 \rightarrow 2$ scalar S-matrix.

Path integrals in the WQFT involve not only the graviton $h_{\mu \nu}(x)$ but also the deflection of each black hole $z_{i}^{\mu}\left(\tau_{i}\right)$, where $x_{i}^{\mu}\left(\tau_{i}\right)=b_{i}^{\mu}+\tau_{i} v_{i}^{\mu}+z_{i}^{\mu}\left(\tau_{i}\right)$ is the full unbound trajectory. We therefore developed a set of Feynman rules to compute expectation values of WQFT operators directly in Fourier space. For the graviton $h_{\mu \nu}(k)$ this of course means momentum space; for the deflection $z_{i}^{\mu}(\omega)$ it instead means energy space. Feynman vertices arising from the purely-gravitational Einstein-Hilbert action conserve four-momentum as usual; vertices arising from a worldline conserve only the energy along that worldline. So even though in these classical calculations we remain at tree level, we see "loop integrals" arising due to the lack of momentum conservation at worldline vertices. These are precisely the integrals one would obtain when working to higher orders in $G$ from an amplitudes perspective. 
Of particular significance was our choice of $i \epsilon$ prescription for the propagators, being either retarded or time-symmetric. For the worldline, using retarded propagators identifies the background parameters $b_{i}^{\mu}$ and $v_{i}^{\mu}$ with the incoming momenta $p_{i}^{\mu}=m_{i} v_{i}^{\mu}$ at $\tau_{i}=-\infty$; time-symmetric propagators identify $\hat{b}_{i}^{\mu}$ and $\hat{v}_{i}^{\mu}$ with the intermediate momenta $\hat{p}_{i}^{\mu}$ at $\tau_{i}=0$. For the gravitons, using Feynman propagators implies a time-reversal symmetric dynamics, hence we are dealing with a purely conservative scattering scenario; retarded propagators are applicable to a radiating system and incorporate radiation-reaction effects.

For the scattering of two non-spinning black holes we considered two $2 \mathrm{PM}$ observables: the radiation, $\left.k^{2}\left\langle h_{\mu \nu}(k)\right\rangle\right|_{k^{2}=0}$, and the impulse on one of the black holes, $\Delta p_{i}^{\mu}=-\left.m_{i} \omega^{2} z_{i}^{\mu}(\omega)\right|_{\omega=0}$, the latter reproducing recent results by Kälin and Porto [65]. We also computed the 3PM three-body radiation, from which 3PM two-body radiation should be obtainable as a special case. We drew tree-level graphs with a single outgoing line - either an outgoing graviton line or an outgoing deflection mode $z^{\mu}$ - and cut the energy/momentum on that line.

The connection with amplitudes is straightforward for radiation. As was observed in ref. [84], at $2 \mathrm{PM}$ order $\left.k^{2}\left\langle h_{\mu \nu}(k)\right\rangle\right|_{k^{2}=0}$ is straightforwardly obtained from a five-point amplitude with two pairs of distinctly flavored external scalars by integrating over internal momenta (with an appropriate integral measure). This formula we derived by integrating out the scalars, leaving the emitted graviton $h_{\mu \nu}(k)$ unaffected. For the deflection $\Delta p_{i}^{\mu}$ there is no clear amplitudes analog; however, from the eikonal phase $\chi$ we derived $\Delta p_{i}^{\mu}$ up to $2 \mathrm{PM}$ by differentiating with respect to the impact parameter $b_{i}^{\mu}$ - a relationship which we showed extends to higher PM orders.

Setting aside the link to amplitudes, the WQFT offers a number of advantages over other methods for obtaining post-Minkowskian (PM) integrands:

1. One has the benefits of a fully diagrammatic approach without needing to take the classical $\hbar \rightarrow 0$ limit.

2. Generating tree-level graphs is simple to achieve algorithmically — for instance using Berends-Giele recursion [141].

3. There is no need to obtain an effective action by integrating out the gravitons.

4. The $i \epsilon$ prescriptions are flexible: with different choices corresponding to retarded or time-symmetric worldline propagators one can identify the background parameters $b_{i}^{\mu}$ and $v_{i}^{\mu}$ with either the incoming or intermediate momenta. Similarly, for the graviton one can incorporate radiation by using retarded propagators.

Our approach complements ongoing work in the PM regime on tackling the integrals required to compute different gravitational observables (see e.g.ref. [139]).

There are numerous opportunities for follow-up work. Of course, we would like to compute observables to higher PM orders: the eikonal phase $\chi$ and deflection $\Delta p_{i}^{\mu}$ at order $4 \mathrm{PM}$ are obvious targets. We also believe that spin can be incorporated in a natural way, by including classical spin vectors $S_{i}^{\mu}$ for each black hole with their own propagators and worldline vertices. In fact, the WQFTs of supersymmetric spinning particles already 
exist $[142,143]$. It will be interesting to see how this relates to ongoing work on amplitudes in higher-spin field theories (see e.g.ref. [95]). Finally, as the link to amplitudes is now readily apparent, it may be worth revisiting the double copy to see how it translates into this new formalism - hopefully clarifying the observed breakdown in ref. [144].

\section{Acknowledgments}

The authors would like to thank Roberto Bonezzi, Gregor Kälin, Rafael Porto and Christian Schubert for interesting and helpful discussions. GM's research is funded by the Deutsche Forschungsgemeinschaft (DFG, German Research Foundation) - Projektnummer 417533893/GRK2575 "Rethinking Quantum Field Theory", the Swedish Research Council under grant 621-2014-5722, the Knut and Alice Wallenberg Foundation under grant KAW 2018.0116, and the Ragnar Söderberg Foundation (Swedish Foundations' Starting Grant).

\section{A Derivation of the momentum space propagator}

In this appendix we further elaborate on the derivation of a momentum space representation of the massive scalar propagator coupled to gravitons, eq. (3.7). The basic ingredient for us to evaluate is

$$
\left\langle\prod_{l=1}^{N} \int_{0}^{s} \mathrm{~d} \tau_{l} e^{i k_{l} \cdot\left(x+\Delta x \frac{\tau_{l}}{s}\right)} \epsilon^{(l) \mu \nu} \tilde{D}_{\mu \nu}^{(l)}\left(x, x^{\prime},\left\{k_{l}\right\}\right)\right\rangle,
$$

with

$$
\begin{aligned}
\tilde{D}_{\mu \nu}^{(l)}\left(x, x^{\prime},\left\{k_{l}\right\}\right)=\left[\left(\frac{\Delta x^{\mu}}{s}+\dot{q}^{\mu}\left(\tau_{l}\right)\right)\right. & \left(\frac{\Delta x^{\nu}}{s}+\dot{q}^{\nu}\left(\tau_{l}\right)\right) \\
& \left.+\mathfrak{a}^{\mu}\left(\tau_{l}\right) \mathfrak{a}^{\nu}\left(\tau_{l}\right)+\mathfrak{b}^{\mu}\left(\tau_{l}\right) \mathfrak{c}^{\nu}\left(\tau_{l}\right)\right] e^{i k_{l} \cdot q\left(\tau_{l}\right)} .
\end{aligned}
$$

We also note that

$$
Z=\int D[q] e^{-i \int_{0}^{s} \mathrm{~d} \tau \frac{1}{4} \dot{q}^{2}}=\frac{1}{(-4 \pi i s)^{D / 2}},
$$

which is the free partition function.

To evaluate the correlation function we use a number of tricks. Firstly, we introduce a scalar function $F$ of the polarization vectors $\epsilon, \alpha, \beta, \gamma$, the latter two of which are anti-commuting:

$$
F(\epsilon, \alpha, \beta, \gamma):=\left\langle\exp \left[\sum_{l=1}^{N} \epsilon_{l} \cdot \dot{q}\left(\tau_{l}\right)+\alpha_{l} \cdot \mathfrak{a}\left(\tau_{l}\right)+\beta_{l} \cdot \mathfrak{b}\left(\tau_{l}\right)+\gamma_{l} \cdot \mathfrak{c}\left(\tau_{l}\right)+i k_{l} \cdot q\left(\tau_{l}\right)\right]\right\rangle .
$$

Then we may write

$$
\begin{aligned}
(\mathrm{A} .1)=\prod_{l=1}^{N} \int_{0}^{s} \mathrm{~d} \tau_{l}\left[\left(\frac{\Delta x_{\mu}}{s}+\partial_{\epsilon_{l}^{\mu}}\right)\right. & \left(\frac{\Delta x_{\nu}}{s}+\partial_{\epsilon_{l}^{\nu}}\right) \\
& \left.+\partial_{\alpha_{l}^{\mu}} \partial_{\alpha_{l}^{\nu}}+\partial_{\beta_{l}^{\mu}} \partial_{\gamma_{\alpha_{l}^{\nu}}}\right]\left.F(\epsilon, \alpha, \beta, \gamma)\right|_{\alpha=\beta=\gamma=\epsilon=0} .
\end{aligned}
$$


To compute $F(\epsilon, \alpha, \beta, \gamma)$ we use the fact that for operators $\mathcal{O}$ linear in quantum fields one has the free-field correlation function relation

$$
\left\langle e^{\mathcal{O}}\right\rangle=\frac{1}{(-4 \pi s i)^{D / 2}} \exp \left[\frac{1}{2}\langle\mathcal{O O}\rangle\right]
$$

Hence we find

$$
F(\epsilon, \alpha, \beta, \gamma)=\frac{1}{(-4 \pi s i)^{D / 2}} \exp \left[\frac{1}{2}\left\langle\sum_{l, l^{\prime}=1}^{N} \mathcal{O}_{l} \mathcal{O}_{l^{\prime}}\right\rangle\right],
$$

with $\mathcal{O}_{l}=\epsilon_{l} \cdot \dot{q}\left(\tau_{l}\right)+\alpha_{l} \cdot \mathfrak{a}\left(\tau_{l}\right)+\beta_{l} \cdot \mathfrak{b}\left(\tau_{l}\right)+\gamma_{l} \cdot \mathfrak{c}\left(\tau_{l}\right)+i k_{l} \cdot q\left(\tau_{l}\right)$. We then compute using eq. (3.6)

$$
\begin{aligned}
i\left\langle\mathcal{O}_{l} \mathcal{O}_{l^{\prime}}\right\rangle= & 2 \delta\left(\tau_{l}-\tau_{l^{\prime}}\right)\left[\epsilon_{l} \cdot \epsilon_{l^{\prime}}+\alpha_{l} \cdot \alpha_{l^{\prime}}-2 \gamma_{l} \cdot \beta_{l^{\prime}}-2 \gamma_{l^{\prime}} \cdot \beta_{l}\right] \\
& -\frac{2}{s}\left[i k_{l} \tau_{l}+\epsilon_{l}\right] \cdot\left[i k_{l^{\prime}} \tau_{l^{\prime}}+\epsilon_{l^{\prime}}\right]+i k_{l} \cdot\left[i k_{l^{\prime}} \tau_{l^{\prime}}+\epsilon_{l^{\prime}}\right]+i k_{l^{\prime}} \cdot\left[i k_{l} \tau_{l}+\epsilon_{l}\right] \\
& -i\left(\epsilon_{l} \cdot k_{l^{\prime}}-\epsilon_{l^{\prime}} \cdot k_{l}\right) \operatorname{sign}\left(\tau_{l}-\tau_{l^{\prime}}\right)+k_{l} \cdot k_{l^{\prime}}\left|\tau_{l}-\tau_{l^{\prime}}\right|
\end{aligned}
$$

For this it is helpful to note the derivatives of the propagator given in eq. (3.6):

$$
\begin{aligned}
\partial_{\tau} \Delta\left(\tau, \tau^{\prime}\right) & =\frac{1}{2} \operatorname{sign}\left(\tau-\tau^{\prime}\right)+\frac{\tau^{\prime}}{s}-\frac{1}{2}, \\
\partial_{\tau^{\prime}} \Delta\left(\tau, \tau^{\prime}\right) & =-\frac{1}{2} \operatorname{sign}\left(\tau-\tau^{\prime}\right)+\frac{\tau}{s}-\frac{1}{2}, \\
\partial_{\tau} \partial_{\tau^{\prime}} \Delta\left(\tau, \tau^{\prime}\right) & =\frac{1}{s}-\delta\left(\tau-\tau^{\prime}\right), \\
\partial_{\tau}^{2} \Delta\left(\tau, \tau^{\prime}\right) & =\delta\left(\tau-\tau^{\prime}\right) .
\end{aligned}
$$

The second trick lies in promoting also the $\Delta x^{\mu} / s$ terms in eq. (A.2) into the exponent of the evaluated $F(\epsilon, \alpha, \beta, \gamma)$ by manually adding $\sum_{l=1}^{N} \epsilon_{l} \cdot \frac{\Delta x}{s}$ to the exponent on the righthand side of eq. (A.5). Then we perform the space-time integrals over $x$ and $x^{\prime}$, giving a total momentum-conserving delta function and a Gaussian integral.

Open Access. This article is distributed under the terms of the Creative Commons Attribution License (CC-BY 4.0), which permits any use, distribution and reproduction in any medium, provided the original author(s) and source are credited.

\section{References}

[1] S. Chandrasekhar, Beauty and the quest for beauty in science, talk given at the International Symposium in Honor of Robert R. Wilson, April 27, Boston, U.S.A. (1979).

[2] L.J. Dixon, Scattering amplitudes: the most perfect microscopic structures in the universe, J. Phys. A 44 (2011) 454001 [arXiv:1105.0771] [InSPIRE].

[3] LIGO SCIENTIFIC, VIRGo collaboration, Observation of gravitational waves from a binary black hole merger, Phys. Rev. Lett. 116 (2016) 061102 [arXiv:1602.03837] [INSPIRE]. 
[4] LIGO Scientific, VIRGO collaboration, GW170817: observation of gravitational waves from a binary neutron star inspiral, Phys. Rev. Lett. 119 (2017) 161101 [arXiv: 1710.05832] [INSPIRE].

[5] LiGO Scientific, ViRgo collaboration, GWTC-1: a gravitational-wave transient catalog of compact binary mergers observed by LIGO and Virgo during the first and second observing runs, Phys. Rev. X 9 (2019) 031040 [arXiv:1811.12907] [INSPIRE].

[6] M. Pürrer and C.-J. Haster, Gravitational waveform accuracy requirements for future ground-based detectors, Phys. Rev. Res. 2 (2020) 023151 [arXiv: 1912.10055] [INSPIRE].

[7] L. Blanchet, Gravitational radiation from post-Newtonian sources and inspiralling compact binaries, Living Rev. Rel. 17 (2014) 2 [arXiv:1310.1528] [INSPIRE].

[8] G. Schäfer and P. Jaranowski, Hamiltonian formulation of general relativity and post-Newtonian dynamics of compact binaries, Living Rev. Rel. 21 (2018) 7 [arXiv: 1805. 07240] [INSPIRE].

[9] T. Futamase and Y. Itoh, The post-Newtonian approximation for relativistic compact binaries, Living Rev. Rel. 10 (2007) 2 [INSPIRE].

[10] M.E. Pati and C.M. Will, Post-Newtonian gravitational radiation and equations of motion via direct integration of the relaxed Einstein equations. 1. Foundations, Phys. Rev. D 62 (2000) 124015 [gr-qc/0007087] [inSPIRE].

[11] L. Bel, T. Damour, N. Deruelle, J. Ibáñez and J. Martin, Poincaré-invariant gravitational field and equations of motion of two pointlike objects: the postlinear approximation of general relativity, Gen. Rel. Grav. 13 (1981) 963 [INSPIRE].

[12] K. Westpfahl, High-speed scattering of charged and uncharged particles in general relativity, Fortsch. Phys. 33 (1985) 417 [InSPIRE].

[13] W.D. Goldberger and I.Z. Rothstein, An effective field theory of gravity for extended objects, Phys. Rev. D 73 (2006) 104029 [hep-th/0409156] [INSPIRE].

[14] W.D. Goldberger and I.Z. Rothstein, Towers of gravitational theories, Gen. Rel. Grav. 38 (2006) 1537 [Int. J. Mod. Phys. D 15 (2006) 2293] [hep-th/0605238] [InSPIRE].

[15] W.D. Goldberger and A. Ross, Gravitational radiative corrections from effective field theory, Phys. Rev. D 81 (2010) 124015 [arXiv:0912.4254] [INSPIRE].

[16] B. Kol and M. Smolkin, Non-relativistic gravitation: from Newton to Einstein and back, Class. Quant. Grav. 25 (2008) 145011 [arXiv:0712.4116] [INSPIRE].

[17] W.D. Goldberger, Les Houches lectures on effective field theories and gravitational radiation, in the proceedings of Les Houches Summer School - Session 86: Particle Physics and Cosmology: The Fabric of Spacetime, July 31-August 25, Les Houches, France (2007), hep-ph/0701129 [INSPIRE].

[18] S. Foffa and R. Sturani, Effective field theory methods to model compact binaries, Class. Quant. Grav. 31 (2014) 043001 [arXiv:1309.3474] [INSPIRE].

[19] I.Z. Rothstein, Progress in effective field theory approach to the binary inspiral problem, Gen. Rel. Grav. 46 (2014) 1726 [inSPIRE].

[20] R.A. Porto, The effective field theorist's approach to gravitational dynamics, Phys. Rept. 633 (2016) 1 [arXiv: 1601.04914] [INSPIRE].

[21] M. Levi, Effective field theories of post-Newtonian gravity: a comprehensive review, Rept. Prog. Phys. 83 (2020) 075901 [arXiv:1807.01699] [INSPIRE]. 
[22] T. Damour, P. Jaranowski and G. Schäfer, Nonlocal-in-time action for the fourth post-Newtonian conservative dynamics of two-body systems, Phys. Rev. D 89 (2014) 064058 [arXiv: 1401.4548] [INSPIRE].

[23] T. Damour, P. Jaranowski and G. Schäfer, Conservative dynamics of two-body systems at the fourth post-Newtonian approximation of general relativity, Phys. Rev. D 93 (2016) 084014 [arXiv: 1601.01283] [INSPIRE].

[24] L. Bernard, L. Blanchet, A. Bohé, G. Faye and S. Marsat, Energy and periastron advance of compact binaries on circular orbits at the fourth post-Newtonian order, Phys. Rev. D 95 (2017) 044026 [arXiv: 1610.07934] [INSPIRE].

[25] S. Foffa, P. Mastrolia, R. Sturani and C. Sturm, Effective field theory approach to the gravitational two-body dynamics, at fourth post-Newtonian order and quintic in the Newton constant, Phys. Rev. D 95 (2017) 104009 [arXiv:1612.00482] [INSPIRE].

[26] T. Damour and P. Jaranowski, Four-loop static contribution to the gravitational interaction potential of two point masses, Phys. Rev. D 95 (2017) 084005 [arXiv:1701.02645] [INSPIRE].

[27] S. Foffa and R. Sturani, Conservative dynamics of binary systems to fourth post-Newtonian order in the EFT approach I: regularized Lagrangian, Phys. Rev. D 100 (2019) 024047 [arXiv: 1903. 05113] [INSPIRE].

[28] S. Foffa, R.A. Porto, I. Rothstein and R. Sturani, Conservative dynamics of binary systems to fourth Post-Newtonian order in the EFT approach II: renormalized Lagrangian, Phys. Rev. D 100 (2019) 024048 [arXiv: 1903.05118] [INSPIRE].

[29] J. Blümlein, A. Maier, P. Marquard and G. Schäfer, Fourth post-Newtonian Hamiltonian dynamics of two-body systems from an effective field theory approach, Nucl. Phys. B $\mathbf{9 5 5}$ (2020) 115041 [arXiv: 2003.01692] [InSPIRE].

[30] R.A. Porto and I.Z. Rothstein, Apparent ambiguities in the post-Newtonian expansion for binary systems, Phys. Rev. D 96 (2017) 024062 [arXiv:1703.06433] [INSPIRE].

[31] T. Marchand, L. Bernard, L. Blanchet and G. Faye, Ambiguity-free completion of the equations of motion of compact binary systems at the fourth post-Newtonian order, Phys. Rev. D 97 (2018) 044023 [arXiv: 1707.09289] [InSPIRE].

[32] C.R. Galley, A.K. Leibovich, R.A. Porto and A. Ross, Tail effect in gravitational radiation reaction: time nonlocality and renormalization group evolution, Phys. Rev. D 93 (2016) 124010 [arXiv: 1511.07379] [INSPIRE].

[33] S. Foffa, P. Mastrolia, R. Sturani, C. Sturm and W.J. Torres Bobadilla, Static two-body potential at fifth post-Newtonian order, Phys. Rev. Lett. 122 (2019) 241605 [arXiv: 1902.10571] [INSPIRE].

[34] J. Blümlein, A. Maier and P. Marquard, Five-loop static contribution to the gravitational interaction potential of two point masses, Phys. Lett. B 800 (2020) 135100 [arXiv: 1902.11180] [INSPIRE].

[35] D. Bini, T. Damour and A. Geralico, Novel approach to binary dynamics: application to the fifth post-Newtonian level, Phys. Rev. Lett. 123 (2019) 231104 [arXiv:1909.02375] [INSPIRE].

[36] J. Blümlein, A. Maier, P. Marquard and G. Schäfer, The fifth-order post-Newtonian Hamiltonian dynamics of two-body systems from an effective field theory approach: potential contributions, arXiv:2010.13672 
[37] J. Blümlein, A. Maier, P. Marquard and G. Schäfer, Testing binary dynamics in gravity at the sixth post-Newtonian level, Phys. Lett. B 807 (2020) 135496 [arXiv:2003.07145] [INSPIRE].

[38] C. Cheung and M.P. Solon, Classical gravitational scattering at $\mathcal{O}\left(G^{3}\right)$ from Feynman diagrams, JHEP 06 (2020) 144 [arXiv: 2003.08351] [INSPIRE].

[39] D. Bini, T. Damour and A. Geralico, Sixth post-Newtonian local-in-time dynamics of binary systems, Phys. Rev. D 102 (2020) 024061 [arXiv:2004.05407] [INSPIRE].

[40] D. Bini, T. Damour and A. Geralico, Binary dynamics at the fifth and fifth-and-a-half post-Newtonian orders, Phys. Rev. D 102 (2020) 024062 [arXiv:2003.11891] [INSPIRE].

[41] D. Bini, T. Damour and A. Geralico, Sixth post-Newtonian nonlocal-in-time dynamics of binary systems, Phys. Rev. D 102 (2020) 084047 [arXiv:2007.11239] [INSPIRE].

[42] D. Bini, T. Damour, A. Geralico, S. Laporta and P. Mastrolia, Gravitational dynamics at $O\left(G^{6}\right)$ : perturbative gravitational scattering meets experimental mathematics, arXiv: 2008.09389 [INSPIRE].

[43] L. Blanchet, B.R. Iyer and B. Joguet, Gravitational waves from inspiralling compact binaries: energy flux to third postNewtonian order, Phys. Rev. D 65 (2002) 064005 [Erratum ibid. 71 (2005) 129903] [gr-qc/0105098] [InSPIRE].

[44] L. Blanchet, T. Damour, G. Esposito-Farese and B.R. Iyer, Gravitational radiation from inspiralling compact binaries completed at the third post-Newtonian order, Phys. Rev. Lett. 93 (2004) 091101 [gr-qc/0406012] [INSPIRE].

[45] L. Blanchet, G. Faye, B.R. Iyer and S. Sinha, The third post-Newtonian gravitational wave polarisations and associated spherical harmonic modes for inspiralling compact binaries in quasi-circular orbits, Class. Quant. Grav. 25 (2008) 165003 [Erratum ibid. 29 (2012) 239501] [arXiv:0802.1249] [INSPIRE].

[46] A.K. Leibovich, N.T. Maia, I.Z. Rothstein and Z. Yang, Second post-Newtonian order radiative dynamics of inspiralling compact binaries in the Effective Field Theory approach, Phys. Rev. D 101 (2020) 084058 [arXiv:1912.12546] [InSPIRE].

[47] R.A. Porto, Post-Newtonian corrections to the motion of spinning bodies in NRGR, Phys. Rev. D 73 (2006) 104031 [gr-qc/0511061] [INSPIRE].

[48] M. Levi and J. Steinhoff, Spinning gravitating objects in the effective field theory in the post-Newtonian scheme, JHEP 09 (2015) 219 [arXiv:1501.04956] [INSPIRE].

[49] M. Levi, A.J. Mcleod and M. Von Hippel, $N^{3} L O$ gravitational spin-orbit coupling at order $G^{4}, \operatorname{arXiv}: 2003.02827$ [INSPIRE].

[50] A. Antonelli, C. Kavanagh, M. Khalil, J. Steinhoff and J. Vines, Gravitational spin-orbit coupling through third-subleading post-Newtonian order: from first-order self-force to arbitrary mass ratios, Phys. Rev. Lett. 125 (2020) 011103 [arXiv:2003.11391] [INSPIRE].

[51] M. Levi and J. Steinhoff, Complete conservative dynamics for inspiralling compact binaries with spins at fourth post-Newtonian order, arXiv:1607.04252 [INSPIRE].

[52] R.A. Porto and I.Z. Rothstein, The hyperfine Einstein-Infeld-Hoffmann potential, Phys. Rev. Lett. 97 (2006) 021101 [gr-qc/0604099] [INSPIRE].

[53] R.A. Porto and I.Z. Rothstein, Spin(1)Spin(2) effects in the motion of inspiralling compact binaries at third order in the post-Newtonian expansion, Phys. Rev. D 78 (2008) 044012 [Erratum ibid. 81 (2010) 029904] [arXiv:0802.0720] [INSPIRE].

[54] M. Levi, Next to leading order gravitational Spin(1)-Spin(2) coupling with Kaluza-Klein reduction, Phys. Rev. D 82 (2010) 064029 [arXiv:0802.1508] [INSPIRE]. 
[55] R.A. Porto and I.Z. Rothstein, Next to leading order Spin(1)-Spin(1) effects in the motion of inspiralling compact binaries, Phys. Rev. D $\mathbf{7 8}$ (2008) 044013 [Erratum ibid. 81 (2010) 029905] [arXiv: 0804.0260] [INSPIRE].

[56] R.A. Porto, Next to leading order spin-orbit effects in the motion of inspiralling compact binaries, Class. Quant. Grav. 27 (2010) 205001 [arXiv:1005.5730] [INSPIRE].

[57] D.L. Perrodin, Subleading spin-orbit correction to the Newtonian potential in effective field theory formalism, in the proceedings of the $12^{\text {th }}$ Marcel Grossmann Meeting on General Relativity, July 20-26, Rio de Janeiro, Brasil (2010) [arXiv: 1005.0634] [INSPIRE].

[58] M. Levi, Next to leading order gravitational spin-orbit coupling in an effective field theory approach, Phys. Rev. D 82 (2010) 104004 [arXiv: 1006.4139] [INSPIRE].

[59] C.K. Mishra, A. Kela, K.G. Arun and G. Faye, Ready-to-use post-Newtonian gravitational waveforms for binary black holes with nonprecessing spins: An update, Phys. Rev. D 93 (2016) 084054 [arXiv: 1601.05588] [INSPIRE].

[60] A. Buonanno, G. Faye and T. Hinderer, Spin effects on gravitational waves from inspiraling compact binaries at second post-Newtonian order, Phys. Rev. D 87 (2013) 044009 [arXiv: 1209.6349] [INSPIRE].

[61] R.A. Porto, A. Ross and I.Z. Rothstein, Spin induced multipole moments for the gravitational wave flux from binary inspirals to third Post-Newtonian order, JCAP 03 (2011) 009 [arXiv: 1007.1312] [inSPIRE].

[62] R.A. Porto, A. Ross and I.Z. Rothstein, Spin induced multipole moments for the gravitational wave amplitude from binary inspirals to 2.5 post-Newtonian order, JCAP 09 (2012) 028 [arXiv: 1203.2962] [INSPIRE].

[63] N.T. Maia, C.R. Galley, A.K. Leibovich and R.A. Porto, Radiation reaction for spinning bodies in effective field theory I: spin-orbit effects, Phys. Rev. D 96 (2017) 084064 [arXiv: 1705. 07934] [INSPIRE].

[64] N.T. Maia, C.R. Galley, A.K. Leibovich and R.A. Porto, Radiation reaction for spinning bodies in effective field theory II: spin-spin effects, Phys. Rev. D 96 (2017) 084065 [arXiv: 1705. 07938] [INSPIRE].

[65] G. Kälin and R.A. Porto, Post-Minkowskian effective field theory for conservative binary dynamics, JHEP 11 (2020) 106 [arXiv: 2006.01184] [INSPIRE].

[66] D. Bini, T. Damour and A. Geralico, Scattering of tidally interacting bodies in post-Minkowskian gravity, Phys. Rev. D 101 (2020) 044039 [arXiv:2001.00352] [InSPIRE].

[67] C. Cheung and M.P. Solon, Tidal effects in the post-Minkowskian expansion, Phys. Rev. Lett. 125 (2020) 191601 [arXiv: 2006.06665] [INSPIRE].

[68] G. Kälin, Z. Liu and R.A. Porto, Conservative tidal effects in compact binary systems to next-to-leading post-Minkowskian order, Phys. Rev. D 102 (2020) 124025 [arXiv: 2008.06047] [INSPIRE].

[69] K. Haddad and A. Helset, Tidal effects in quantum field theory, JHEP 12 (2020) 024 [arXiv: 2008.04920] [INSPIRE].

[70] G. Kälin, Z. Liu and R.A. Porto, Conservative dynamics of binary systems to third post-Minkowskian order from the effective field theory approach, Phys. Rev. Lett. 125 (2020) 261103 [arXiv: 2007.04977] [inSPIRE].

[71] T. Ledvinka, G. Schaefer and J. Bicak, Relativistic closed-form Hamiltonian for many-body gravitating systems in the post-Minkowskian approximation, Phys. Rev. Lett. 100 (2008) 251101 [arXiv: 0807.0214] [INSPIRE]. 
[72] T. Damour, Gravitational scattering, post-Minkowskian approximation and Effective One-Body theory, Phys. Rev. D 94 (2016) 104015 [arXiv: 1609. 00354] [InSPIRE].

[73] L. Blanchet and A.S. Fokas, Equations of motion of self-gravitating $N$-body systems in the first post-Minkowskian approximation, Phys. Rev. D 98 (2018) 084005 [arXiv:1806.08347] [INSPIRE].

[74] S.J. Kovacs and K.S. Thorne, The generation of gravitational waves. III. Derivation of bremsstrahlung formulae, Astrophys. J. 217 (1977) 252.

[75] S.J. Kovacs and K.S. Thorne, The generation of gravitational waves. IV. Bremsstrahlung, Astrophys. J. 224 (1978) 62.

[76] D. Bini and T. Damour, Gravitational spin-orbit coupling in binary systems, post-Minkowskian approximation and effective one-body theory, Phys. Rev. D 96 (2017) 104038 [arXiv: 1709.00590] [INSPIRE].

[77] J. Vines, Scattering of two spinning black holes in post-Minkowskian gravity, to all orders in spin, and effective-one-body mappings, Class. Quant. Grav. 35 (2018) 084002 [arXiv:1709.06016] [INSPIRE].

[78] D. Bini and T. Damour, Gravitational spin-orbit coupling in binary systems at the second post-Minkowskian approximation, Phys. Rev. D 98 (2018) 044036 [arXiv:1805.10809] [INSPIRE].

[79] Y. Iwasaki, Quantum theory of gravitation vs. classical theory - Fourth-order potential, Prog. Theor. Phys. 46 (1971) 1587 [inSPIRE].

[80] M.J. Duff, Quantum tree graphs and the Schwarzschild solution, Phys. Rev. D 7 (1973) 2317 [INSPIRE].

[81] B.R. Holstein and J.F. Donoghue, Classical physics and quantum loops, Phys. Rev. Lett. 93 (2004) 201602 [hep-th/0405239] [INSPIRE].

[82] D. Neill and I.Z. Rothstein, Classical space-times from the S-matrix, Nucl. Phys. B $\mathbf{8 7 7}$ (2013) 177 [arXiv: 1304.7263] [INSPIRE].

[83] N.E.J. Bjerrum-Bohr, J.F. Donoghue and P. Vanhove, On-shell techniques and universal results in quantum gravity, JHEP 02 (2014) 111 [arXiv: 1309.0804] [INSPIRE].

[84] A. Luna, I. Nicholson, D. O'Connell and C.D. White, Inelastic black hole scattering from charged scalar amplitudes, JHEP 03 (2018) 044 [arXiv:1711.03901] [INSPIRE].

[85] N.E.J. Bjerrum-Bohr, P.H. Damgaard, G. Festuccia, L. Planté and P. Vanhove, General relativity from scattering amplitudes, Phys. Rev. Lett. 121 (2018) 171601 [arXiv: 1806.04920] [INSPIRE].

[86] D.A. Kosower, B. Maybee and D. O'Connell, Amplitudes, observables, and classical scattering, JHEP 02 (2019) 137 [arXiv:1811.10950] [INSPIRE].

[87] C. Cheung, I.Z. Rothstein and M.P. Solon, From scattering amplitudes to classical potentials in the post-Minkowskian expansion, Phys. Rev. Lett. 121 (2018) 251101 [arXiv: 1808.02489] [INSPIRE].

[88] A. Cristofoli, N.E.J. Bjerrum-Bohr, P.H. Damgaard and P. Vanhove, Post-Minkowskian Hamiltonians in general relativity, Phys. Rev. D 100 (2019) 084040 [arXiv:1906.01579] [INSPIRE].

[89] Z. Bern, C. Cheung, R. Roiban, C.-H. Shen, M.P. Solon and M. Zeng, Scattering amplitudes and the conservative Hamiltonian for binary systems at third post-Minkowskian order, Phys. Rev. Lett. 122 (2019) 201603 [arXiv:1901.04424] [INSPIRE]. 
[90] Z. Bern, C. Cheung, R. Roiban, C.-H. Shen, M.P. Solon and M. Zeng, Black hole binary dynamics from the double copy and effective theory, JHEP 10 (2019) 206 [arXiv: 1908.01493] [INSPIRE].

[91] V. Vaidya, Gravitational spin Hamiltonians from the S matrix, Phys. Rev. D 91 (2015) 024017 [arXiv: 1410.5348] [INSPIRE].

[92] A. Guevara, A. Ochirov and J. Vines, Scattering of spinning black holes from exponentiated soft factors, JHEP 09 (2019) 056 [arXiv:1812.06895] [INSPIRE].

[93] A. Guevara, A. Ochirov and J. Vines, Black-hole scattering with general spin directions from minimal-coupling amplitudes, Phys. Rev. D 100 (2019) 104024 [arXiv:1906.10071] [INSPIRE].

[94] B. Maybee, D. O'Connell and J. Vines, Observables and amplitudes for spinning particles and black holes, JHEP 12 (2019) 156 [arXiv:1906.09260] [INSPIRE].

[95] Z. Bern, A. Luna, R. Roiban, C.-H. Shen and M. Zeng, Spinning black hole binary dynamics, scattering amplitudes and effective field theory, arXiv:2005.03071 [INSPIRE].

[96] P.H. Damgaard, K. Haddad and A. Helset, Heavy black hole effective theory, JHEP 11 (2019) 070 [arXiv: 1908.10308] [INSPIRE].

[97] R. Aoude, K. Haddad and A. Helset, On-shell heavy particle effective theories, JHEP 05 (2020) 051 [arXiv: 2001.09164] [InSPIRE].

[98] T. Damour, Classical and quantum scattering in post-Minkowskian gravity, Phys. Rev. D 102 (2020) 024060 [arXiv: 1912.02139] [INSPIRE].

[99] T. Damour, High-energy gravitational scattering and the general relativistic two-body problem, Phys. Rev. D 97 (2018) 044038 [arXiv:1710.10599] [INSPIRE].

[100] G. Kälin and R.A. Porto, From boundary data to bound states, JHEP 01 (2020) 072 [arXiv: 1910.03008] [INSPIRE].

[101] G. Kälin and R.A. Porto, From boundary data to bound states. Part II. Scattering angle to dynamical invariants (with twist), JHEP 02 (2020) 120 [arXiv:1911.09130] [INSPIRE].

[102] F. Bastianelli, O. Corradini and P. van Nieuwenhuizen, Dimensional regularization of the path integral in curved space on an infinite time interval, Phys. Lett. B 490 (2000) 154 [hep-th/0007105] [INSPIRE].

[103] F. Bastianelli and A. Zirotti, Worldline formalism in a gravitational background, Nucl. Phys. B 642 (2002) 372 [hep-th/0205182] [INSPIRE].

[104] M. Fabbrichesi, R. Pettorino, G. Veneziano and G.A. Vilkovisky, Planckian energy scattering and surface terms in the gravitational action, Nucl. Phys. B 419 (1994) 147 [hep-th/9309037] [INSPIRE].

[105] D. Amati, M. Ciafaloni and G. Veneziano, Can space-time be probed below the string size?, Phys. Lett. B 216 (1989) 41 [inSPIRE].

[106] D. Amati, M. Ciafaloni and G. Veneziano, Higher order gravitational deflection and soft Bremsstrahlung in planckian energy superstring collisions, Nucl. Phys. B 347 (1990) 550 [INSPIRE].

[107] R.P. Feynman, Mathematical formulation of the quantum theory of electromagnetic interaction, Phys. Rev. 80 (1950) 440 [InSPIRE].

[108] M.D. Schwartz, Quantum field theory and the standard model, Cambridge University Press, Cambridge U.K. (2014). 
[109] M.J. Strassler, Field theory without Feynman diagrams: one loop effective actions, Nucl. Phys. B 385 (1992) 145 [hep-ph/9205205] [INSPIRE].

[110] Z. Bern and D.A. Kosower, The computation of loop amplitudes in gauge theories, Nucl. Phys. B 379 (1992) 451 [INSPIRE].

[111] C. Schubert, Perturbative quantum field theory in the string inspired formalism, Phys. Rept. 355 (2001) 73 [hep-th/0101036] [INSPIRE].

[112] J.P. Edwards and C. Schubert, Quantum mechanical path integrals in the first quantised approach to quantum field theory, arXiv:1912.10004 [INSPIRE].

[113] B.S. DeWitt, Dynamical theory in curved spaces. 1. A review of the classical and quantum action principles, Rev. Mod. Phys. 29 (1957) 377 [INSPIRE].

[114] L. Parker, Path integrals for a particle in curved space, Phys. Rev. D 19 (1979) 438 [INSPIRE].

[115] J.D. Bekenstein and L. Parker, Path integral evaluation of Feynman propagator in curved space-time, Phys. Rev. D 23 (1981) 2850 [InSPIRE].

[116] F. Bastianelli and P. van Nieuwenhuizen, Trace anomalies from quantum mechanics, Nucl. Phys. B 389 (1993) 53 [hep-th/9208059] [INSPIRE].

[117] N. Ahmadiniaz, F.M. Balli, O. Corradini, J.M. Dávila and C. Schubert, Compton-like scattering of a scalar particle with $N$ photons and one graviton, Nucl. Phys. B 950 (2020) 114877 [arXiv: 1908.03425] [INSPIRE].

[118] K. Daikouji, M. Shino and Y. Sumino, Bern-Kosower rule for scalar QED, Phys. Rev. D 53 (1996) 4598 [hep-ph/9508377] [INSPIRE].

[119] N. Ahmadiniaz, A. Bashir and C. Schubert, Multiphoton amplitudes and generalized Landau-Khalatnikov-Fradkin transformation in scalar QED, Phys. Rev. D 93 (2016) 045023 [arXiv: 1511.05087] [INSPIRE].

[120] N. Ahmadiniaz, F. Bastianelli and O. Corradini, Dressed scalar propagator in a non-Abelian background from the worldline formalism, Phys. Rev. D 93 (2016) 025035 [Addendum ibid. 93 (2016) 049904] [arXiv: 1508.05144] [INSPIRE].

[121] D. Bonocore, Asymptotic dynamics on the worldline for spinning particles, arXiv: 2009.07863 [INSPIRE].

[122] D. Amati, M. Ciafaloni and G. Veneziano, Superstring collisions at Planckian energies, Phys. Lett. B 197 (1987) 81 [INSPIRE].

[123] T. Damour, Radiative contribution to classical gravitational scattering at the third order in G, Phys. Rev. D 102 (2020) 124008 [arXiv:2010.01641] [inSPIRE].

[124] P. Di Vecchia, C. Heissenberg, R. Russo and G. Veneziano, Universality of ultra-relativistic gravitational scattering, Phys. Lett. B 811 (2020) 135924 [arXiv:2008.12743] [INSPIRE].

[125] N. Arkani-Hamed, Y.-t. Huang and D. O'Connell, Kerr black holes as elementary particles, JHEP 01 (2020) 046 [arXiv: 1906.10100] [INSPIRE].

[126] R. Monteiro, D. O'Connell and C.D. White, Black holes and the double copy, JHEP 12 (2014) 056 [arXiv: 1410.0239] [INSPIRE].

[127] R. Monteiro, D. O'Connell and C.D. White, Gravity as a double copy of gauge theory: from amplitudes to black holes, Int. J. Mod. Phys. D 24 (2015) 1542008 [inSPIRE].

[128] A. Luna, R. Monteiro, D. O'Connell and C.D. White, The classical double copy for Taub-NUT spacetime, Phys. Lett. B $\mathbf{7 5 0}$ (2015) 272 [arXiv:1507.01869] [INSPIRE]. 
[129] W.D. Goldberger and A.K. Ridgway, Radiation and the classical double copy for color charges, Phys. Rev. D 95 (2017) 125010 [arXiv:1611.03493] [InSPIRE].

[130] C.R. Galley and M. Tiglio, Radiation reaction and gravitational waves in the effective field theory approach, Phys. Rev. D 79 (2009) 124027 [arXiv: 0903.1122] [INSPIRE].

[131] C.R. Galley, Classical mechanics of nonconservative systems, Phys. Rev. Lett. 110 (2013) 174301 [arXiv: 1210.2745] [INSPIRE].

[132] A.P. Saha, B. Sahoo and A. Sen, Proof of the classical soft graviton theorem in $D=4$, JHEP 06 (2020) 153 [arXiv: 1912.06413] [INSPIRE].

[133] B. Sahoo, Classical sub-subleading soft photon and soft graviton theorems in four spacetime dimensions, JHEP 12 (2020) 070 [arXiv:2008.04376] [INSPIRE].

[134] B. Sahoo and A. Sen, Classical and quantum results on logarithmic terms in the soft theorem in four dimensions, JHEP 02 (2019) 086 [arXiv: 1808.03288] [INSPIRE].

[135] C.-H. Shen, Gravitational radiation from color-kinematics duality, JHEP 11 (2018) 162 [arXiv: 1806. 07388] [INSPIRE].

[136] J. M. Martín-García, xPerm: fast index canonicalization for tensor computer algebra, Comp. Phys. Commun. 179 (2008) 597 [arXiv:0803.0862].

[137] P. Di Vecchia, S.G. Naculich, R. Russo, G. Veneziano and C.D. White, A tale of two exponentiations in $\mathcal{N}=8$ supergravity at subleading level, JHEP 03 (2020) 173 [arXiv: 1911.11716] [INSPIRE].

[138] A. Antonelli, C. Kavanagh, M. Khalil, J. Steinhoff and J. Vines, Gravitational spin-orbit and aligned spin $_{1}-$ spin $_{2}$ couplings through third-subleading post-Newtonian orders, Phys. Rev. D 102 (2020) 124024 [arXiv:2010.02018] [INSPIRE].

[139] J. Parra-Martinez, M.S. Ruf and M. Zeng, Extremal black hole scattering at $\mathcal{O}\left(G^{3}\right)$ : graviton dominance, eikonal exponentiation, and differential equations, JHEP 11 (2020) 023 [arXiv: 2005. 04236] [INSPIRE].

[140] J. Vines, J. Steinhoff and A. Buonanno, Spinning-black-hole scattering and the test-black-hole limit at second post-Minkowskian order, Phys. Rev. D 99 (2019) 064054 [arXiv:1812.00956] [INSPIRE].

[141] F.A. Berends and W.T. Giele, Recursive calculations for processes with n gluons, Nucl. Phys. B 306 (1988) 759 [INSPIRE].

[142] F. Bastianelli, R. Bonezzi, O. Corradini and E. Latini, One-loop quantum gravity from the $\mathcal{N}=4$ spinning particle, JHEP 11 (2019) 124 [arXiv:1909.05750] [INSPIRE].

[143] R. Bonezzi, A. Meyer and I. Sachs, A Worldline Theory for Supergravity, JHEP 06 (2020) 103 [arXiv: 2004.06129] [inSPIRE].

[144] J. Plefka, C. Shi, J. Steinhoff and T. Wang, Breakdown of the classical double copy for the effective action of dilaton-gravity at NNLO, Phys. Rev. D 100 (2019) 086006 [arXiv: 1906.05875] [INSPIRE]. 